\title{
The variance theory of the mirror effect in recognition memory
}

\author{
SVERKER SIKSTRÖM \\ Stockholm University, Stockholm, Sweden
}

\begin{abstract}
The mirror effect refers to a rather general empirical finding showing that, for two classes of stimuli, the class with the higher hit rates also has a lower false alarm rate. In this article, a parsimonious theory is proposed to account for the mirror effect regarding, specifically, high- and low-frequency items and the associated receiver-operating curves. The theory is implemented in a recurrent network in which one layer represents items and the other represents contexts. It is shown that the frequency mirror effect is found in this simple network if the decision is based on counting the number of active nodes in such a way that performance is optimal or near optimal. The optimal performance requires that the number of active nodes is low, only nodes active in the encoded representation are counted, the activation threshold is set between the old and the new distributions, and normalization is based on the variance of the input. Owing to the interference caused by encoding the to-be-recognized item in several preexperimental contexts, the variance of the input to the context layer is greater for highthan for low-frequency items, which yields lower hit rates and higher false alarm rates for high- than for low-frequency items. Although initially the theory was proposed to account for the mirror effect with respect to word frequency, subsequent simulations have shown that the theory also accounts for strength-based mirror effects within a list and between lists. In this case, consistent with experimental data, the variance theory suggests that focusing attention to the more difficult class within a list affects the hit rate, but not the false alarm rate and not the standard deviations of the underlying density, leading to no mirror effect.
\end{abstract}

The mirror effect refers to a general class of empirical phenomena regarding the order found in hit and false alarm rates for two classes of studied items, when one of the two classes is easier to recognize (A) and produces a larger $d^{\prime}$ than does the other (B). For instance, in the case of a free-choice yes-no recognition task, the mirror effect for new $(\mathrm{N})$ or old $(\mathrm{O})$ items is seen in the following order in terms of increasing probabilities $(P)$ of yes responses: easy new, difficult new, difficult old, and easy old $[P(\mathrm{AN})<P(\mathrm{BN})<P(\mathrm{BO})<P(\mathrm{AO})]$. The phenomenon is named the mirror effect because the order for the old items is the reverse of the order of the new items.

The mirror effect has been studied extensively in different contexts and has been shown to be a rather universal, robust, and basic recognition memory phenomenon. It has been found for many classes of stimuli that differ in several stimulus dimensions (e.g., word frequency, concreteness, material, and strength; for more detailed reviews, see Glanzer \& Adams, 1985, and Glanzer, Adams,

This research was supported by Grant APA 146 from NSERC to Ben Murdock and by a postdoctoral grant from STINT to the author. I thank Ben Murdock for insightful advice and careful comments during the writing of this paper. I also thank Tim Curran, Kisok Kim, Matthew Duncan, Dave Smith, and Carola Åberg for comments on the paper. A special thanks to Shu-Chen Li for helpful comments and support during the last stage of the revising process. Correspondence concerning this article should be addressed to S. Sikström, Department of Psychology, Stockholm University, S-10691 Stockholm, Sweden (e-mail: sverker@ psych.utoronto.ca).
\& Kim, 1993). Furthermore, a few quantitative theories have also been proposed to explain the psychological mechanisms causing the mirror effect (e.g., Glanzer et al., 1993; Greene, 1996; McClelland \& Chappell, 1998; Shiffrin \& Steyvers, 1997). However, as will be discussed later, these current models do not predict some aspects of the mirror effect. This paper presents a new theory whose aim is to extend the previous models in order to overcome these challenges.

\section{Different Facets of the Mirror Effect}

First, I review a few major empirical findings regarding the mirror effect. For instance, Glanzer and Adams (1985) found a material-based mirror effect with respect to concrete versus abstract words and pictures versus words. In terms of the concreteness-based mirror effect, the hit rates of the concrete words are larger, but also the false alarm rates are lower. With respect to pictures versus words, the $d^{\prime}$ 's and the hit rates of the pictures are larger and higher, but also the false alarm rates are lower. The frequency-based mirror effect is, however, most commonly studied. In this case, low-frequency words are easier to recognize and produce lager $d^{\prime}$ s, and the mirror effect is thus as follows: Hit rates are higher for low-frequency words than for high-frequency words, whereas the opposite is true for false alarm rates. The underlying density distributions for old and new high- and low-frequency words corresponding to this phenomenon are as shown in Figure 1. 


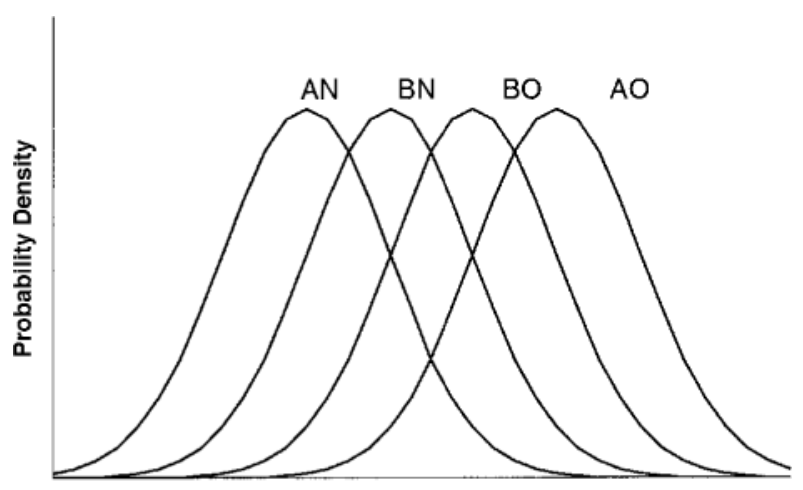

Decision Axis

Figure 1. Hypothetical underlying distributions along the decision axis for low-frequency new items (AN), high-frequency new items (BN), high-frequency old items (BO), and lowfrequency old items (AO). For illustrative purposes, the variances are plotted in this graph to be equal. The horizontal axis shows the recognition strength that the decision is made on, and the vertical axis the density of this recognition strength.

The mirror effect has also been studied with twoalternative forced-choice recognition tests. There are several possible two-alternative comparisons between old and new items. For instance, when high- and low-frequency (better recognized) words are used, there are four standard pairings of old and new by high- and low-frequency comparisons, plus the two null-choice conditions (Glanzer $\&$ Bowles, 1976) in which either a pair of new items or a pair of old items are compared. According to the mirror effect, the order for the four standard pairs is

$$
P(\mathrm{BO}, \mathrm{BN})<P(\mathrm{AO}, \mathrm{BN}), P(\mathrm{BO}, \mathrm{AN})<P(\mathrm{AO}, \mathrm{AN}),
$$

where $P(\mathrm{O}, \mathrm{N})$ is the probability of choosing old over new. Regarding the null-choice conditions, the mirror effect suggests that

$$
P(\mathrm{BN}, \mathrm{AN})>.50, P(\mathrm{AO}, \mathrm{BO})>.50 \text {. }
$$

Another general finding of the mirror effect is that strength manipulations that affect the positions of the new distribution also affect the old distribution when the manipulations are made between conditions (Glanzer et al., 1993). Examples of frequently used experimental manipulations of strength are the duration of study time, encoding conditions, and speed versus accuracy instructions. These experimental manipulations affect both the new and the old distributions by moving the distributions closer together, an effect known as concentering, or by moving the distributions apart, an effect known as dispersion. However, if these strength manipulations are applied differently to items within a condition or in a single list, quite different results are found. For instance, recently Stretch and Wixted (1998a, 1998b) differentially strengthened high-frequency words by presenting them with a higher presentation frequency than that for lowfrequency words. This manipulation affected the hit rate of the high-frequency words, whereas false alarm rates were largely unaffected. Thus, this manipulation did not show a standard mirror effect.

In addition to the basic data with respect to hit and false alarm rates, data on $z$-transformed (or normalized) receiveroperating characteristic curves (z-ROCs) represent another regularity of the distributions underlying the mirror effect. The $z$-ROC-curves are obtained by plotting the $z$-transformation of hit rates against the $z$-transformation of false alarm rates while varying the recognition criterion. According to signal detection theory, the slope of this curve is equal to the standard deviation of the newitem distribution divided by the standard deviation of the old-item distribution. Data on the mirror effect suggests that the standard deviations of the underlying item distributions are symmetrical with respect to the classes (Glanzer \& Adams, 1990; Glanzer et al., 1993). The standard deviation $(\sigma)$ of the recognition strength of the olditem distribution for the difficult class is smaller than the old-item distribution for the easier class [i.e., $\sigma(\mathrm{BO})<$ $\sigma(\mathrm{AO})]$. The standard deviation of the new-item distribution for the difficult class is smaller than the standard deviation for the easier class [i.e., $\sigma(\mathrm{BN})<\sigma(\mathrm{AN})$ ]. However, this symmetry is tilted, so that the standard deviation of the new distribution is smaller than the standard deviation of the old distribution [i.e., $\sigma(\mathrm{N})<\sigma(\mathrm{O})$; Ratcliff, Sheu, \& Gronlund, 1992].

\section{Previous Theories of the Mirror Effect}

In addition to its empirical generality, the mirror effect is an important benchmark phenomenon because it has challenged many global matching memory models, such as SAM (Gillund \& Shiffrin, 1984), MINERVA (Hintzman, 1988), CHARM (Metcalfe, 1982), TODAM (Murdock, 1982), and the matrix model (Humphreys, Bain, \& Pike, 1989). The main limitation of the global memory models in capturing the mirror effect arises from their lack of mechanisms for predicting differential false alarm rates for $\mathrm{BN}$ and $\mathrm{AN}$ items, given that, by definition, these are the new items and neither class of items is presented during the study phase. Confronted with the limitations of the global memory models, the mirror effect had fostered the development of several recent theories to account for the psychological mechanisms that give rise to the phenomenon itself, but as will become clear later as these theories are presented, many controversies still remain.

Response strategy account. For instance, Greene (1996) proposed a response-strategy-based account for the mirror effect regarding order and associative information. Specifically, it was suggested that if subjects equate the number of responses made to each class of words (assuming that the number of lures and targets are approximately equal), this simple response strategy would produce response distributions that give rise to the mirror effect. However, Glanzer, Kisok, and Adams (1998; see also Murdock, 1998) argued against this account by showing that the mirror effect is still present in the absence of a distinctive set of data and in the absence of response equalization. Furthermore, Greene's account pre- 
dicts a mirror effect when subjects are made to focus the attention on the more difficult class. However, increasing the number of hits for the difficult class actually diminishes the false alarm rate. Thus, the response strategy account is at odds with the experimental data.

Attention-likelihood theory. Another attempt to account for the mirror effect is the attention-likelihood theory (Glanzer \& Adams, 1990; Glanzer et al., 1993). This theory says that the difference between $\mathrm{BO}$ and $\mathrm{AO}$ occurs because subjects pay more attention to items in Class A (i.e., the better-recognized class) than to the items in Class B. However, the four distributions (i.e., AN, $\mathrm{BN}, \mathrm{BO}$, and $\mathrm{AO}$ ) along the decision axis reflecting the mirror effect occur mainly because subjects transform the recognition strength of the items to log-likelihood ratios and use that as the basis for their decision.

More specifically, the attention-likelihood theory consists of the following assumptions. (1) Stimuli consist of $N$ number of features. The features are either marked or unmarked. A marked feature indicates that it was in the study list. (2) Some proportion $p$ (new) of features are already marked in a new stimulus, which reflects the noise level, with the rationale that features in a new item (which is not studied during encoding) should be marked because of the random noise in the decision process. (3) Different classes of stimuli $(i)$ evoke different amounts of attention $[n(i)]$. (4) During study, $n(i)$ features are sampled and marked. The proportion of feature sampled for a given stimulus is $n(i) / N$. Given that when the noise level is not zero, some proportion of the new items will also be marked, the probability that a feature is marked becomes

$$
p(i, \text { old })=p(\text { new })+\frac{n(i)}{N}[1-p(\text { new })] .
$$

(5) During recognition, subjects randomly sample a set of $n(i)$ number of features. Given that the sampling is independent of whether the features were marked at study, the number of marked features is thus binomially distributed, with parameters $n(i)$ and $p(i$,old) for old stimuli and $n(i)$ and $p$ (new) for new stimuli. (6) During test, subjects count the number of marked features $(x)$. They note the difference between the sample size and the number of marked features [i.e., $n(i)-x$ ]. Yes-no decisions are then based on the log-likelihood ratio given a class $[\lambda(x \mid i)]$, and it is defined jointly by $x, n(i), p($ new $)$, and $p(i$, old $)$ :

$$
\lambda(x \mid i)=\ln \left[\frac{p(i, \text { old })^{x}\left(1-p(i, \text { old })^{n(i)-x}\right.}{p(\text { new })^{x}\left(1-p(\text { new })^{n(i)-x}\right.}\right] .
$$

Therefore, in contrast to strength theories, which include most of the global memory models, the recognition decision is made along the log-likelihood dimension, rather than the dimension of item strength.

In addition to the mirror effect with respect to the ordering of response distributions of different classes of stimuli, the attention-likelihood theory predicts the following inequalities for the slopes (defined by the ratios between the standard deviations of the response distributions for different stimulus classes) of the $z$-ROC curves:

$$
\begin{aligned}
& \sigma(\mathrm{AO}) / \sigma(\mathrm{BN})<\sigma(\mathrm{AO}) / \sigma(\mathrm{AN}) \\
& <\sigma(\mathrm{BO}) / \sigma(\mathrm{BN})<\sigma(\mathrm{BO}) / \sigma(\mathrm{AN}) .
\end{aligned}
$$

This prediction constrains the standard deviation of the distribution of the difficult class to be smaller than the standard deviation of the distribution of the easy class [i.e., $\sigma(\mathrm{BN})<\sigma(\mathrm{AN})$ and $\sigma(\mathrm{BO})<\sigma(\mathrm{AO})]$. However, it does not constrain the standard deviation of the new distribution to be smaller than the standard deviation of the old distribution [i.e., $\sigma(\mathrm{BO})$ may be smaller than $\sigma(\mathrm{BN})$ ]. There are 24 possible ways (i.e., $4 !=24$ ) to order the four standard deviations. Equation 2 allows 3 out of the 24 orderings - namely, $\sigma(\mathrm{BO})<\sigma(\mathrm{BN})<\sigma(\mathrm{AN})<$ $\sigma(\mathrm{AO}), \sigma(\mathrm{BN})<\sigma(\mathrm{AN})<\sigma(\mathrm{BO})<\sigma(\mathrm{AO})$, and $\sigma(\mathrm{BN})<$ $\sigma(\mathrm{BO})<\sigma(\mathrm{AN})<\sigma(\mathrm{AO})$. However, not all 3 orderings are found in the empirical data. As will be presented later, the variance theory proposed in this paper is more constraining than the attention-likelihood theory, and it permits only those 2 of the orderings that are in line with the empirical data. The attention-likelihood theory has recently inspired at least two models, which also are based on log-likelihoodratios-namely, the subjective-likelihood account of recognition memory (McClelland \& Chappell, 1998) and REM (Shiffrin \& Steyvers, 1997). Below, they are presented in turn.

The subjective-likelihood model. The subjectivelikelihood model for recognition memory (McClelland \& Chappell, 1998) has been applied to the mirror effect with respect to list length, list strength, $z$-ROC curves, and other recognition phenomena. A major difference between McClelland and Chappell's approach and the attentionlikelihood theory is that the subjective-likelihoodaccount uses one detector for each old and each new item. These detectors contrast the probability that a to-be-recognized item is an old item with the probability that it is a new item to form the odds ratio. The model makes an old decision if at least one of the logarithms of the odds ratio is larger than a certain fixed criterion; otherwise, a new decision is made. Differing from the attention-likelihood theory, the odds ratio in the subjective-likelihood model is calculated on estimates of the probabilities, rather than on the true values that a recognition system (or subject) might not have access to, but was nonetheless used in Glanzer et al.'s (1993) model.

The usage of a limited number of detectors, one for each item, in the subjective-likelihood theory is a central mechanism, used to account for several phenomena. For example, the strength-based mirror effect is accounted for by the assumption that detectors for strong items, when strengthened, are less likely to be activated by new items during recognition, which then lowers the false alarm rate. This mechanism works when the number of targets is reasonably large in relation to the number of potential distractors, which was assumed in McClelland and Chappell's (1998) simulations. However, in reality, the num- 
ber of targets in a typical recognition test (for example 50 ) is negligible in comparison with the number of possible distractor words (the number of words in the subject's vocabulary-e.g., 20,000). Thus, the subjective-likelihood account for the list-strength effect may encounter a problem when the number of detectors associated to each distractor increases to a more realistic number. A similar problem may also be apparent with respect to the listlength effect, which is accounted for by the assumption that the number of detectors is proportional to the list length. Arguably, the subjective-likelihood theory would not account for the list-length effect, given a more plausible assumption that the number of detectors is related to the size of the subject's vocabulary.

REM. REM (Shiffrin \& Steyvers, 1997) is another model whose aim is to account for the mirror effect, ROC curves, list strength, and other phenomena in recognition and recall. Similar to the subjective-likelihood theory, REM is based on likelihood ratios and uses noisy vectorbased representations in memory. Although REM also uses likelihood ratios, REM differs in the sense that it uses true probabilities in calculating the likelihood ratios, whereas the subjective-likelihood theory uses estimates. Furthermore, in REM the value assigned to the model's representation on a particular feature dimension is determined when the dimension is sampled the first time. In the subjective-likelihood theory, the representations of the items are refined successively over presentations.

In REM, several factors are combined together to produce the frequency-based mirror effect. (1) The likelihood ratios are assumed to be smaller for old high-frequency words, because high-frequency features are less diagnostic. (2) This factor is larger than the compensating factor that high-frequency words have slightly more matching feature values, because errors in storage tend to produce common values, increasing the probability of accidentally matching a high-frequency feature value. (3) New high-frequency words have a slight increase in the number of chance matches, which increases the likelihood ratio.

Limitations of current theories. Although these theories account for several aspects of the data regarding the mirror effect, they have been subjected to a few general criticisms. Perhaps the most obvious problem with these models is that they predict that strengthening of a class yields a mirror effect. Although this prediction is supported by data in studies in which the strength manipulations were applied between conditions (Glanzer et al., 1993), it is certainly inconsistent with the data when the strength manipulations were applied within conditions (Stretch \& Wixted, 1998a; see also Stretch \& Wixted, 1998b).

In addition, there are a few other more specific criticisms about these theories. Here are some problems regarding the attention-likelihood theory. First, calculating the log-likelihood ratios requires knowledge of the class ( $\lambda$ dependent on $i$ ). Thus, it is necessary to classify the to-be-recognized stimuli into distinct categories $(i)$ or, at least, to estimate the stimuli along some dimension, such as frequency. Glanzer et al. (1993) noted that the attentionlikelihood theory predicts the mirror effect even though the estimates of $p(i$, old $)$ are not accurate-for example, when this probability is set to the average of the two classes. Thus, the estimates of $p(i$,old $)$ are not critical to the predictions. However it is necessary to estimate the number of features sampled at recognition $[n(i)]$ in Equation 1 to make the correct predictions, and this process requires knowledge of the class of stimuli. Second, knowledge of several variables is required. Depending on the classification, the attention paid to this category $[n(i)]$ must be estimated. The probability that a feature in a new item is marked must also be estimated. Third, the attention-likelihood theory involves several steps of complex calculation. Although this may not be the reason for dismissing the theory (see Murdock, 1998, for a discussion of this topic), it would be preferable to have a simpler account. Given that the subjective-likelihood account (McClelland \& Chappell, 1998) and REM, like the attention-likelihood theory (Shiffrin \& Steyvers, 1997), are also based at their cores on variations in log-likelihood ratios, these criticisms would also apply to them.

\section{Research Goal and Organization of the Paper}

Given the limitations of current theories, the purpose of this paper is to propose a new account of the mirror effect that can avoid most of these criticisms. The theory is proposed specifically for the frequency-based mirror effect, but it also accounts for the strength-based mirror effect within a list, the strength-based mirror effect between lists, $z$-ROC curves associated with the mirror effect, and the list-length effect. The paper is organized as follows. First, a brief overview of the theory is presented, which is then followed by an in-depth presentation. Second, the theory is implemented in a connectionist model of memory previously developed by the author (i.e., TECO, the target, event, cue, and object theory; Sikström, 1996a, 1996b). Third, mechanisms of the theory responsible for capturing the mirror effect are presented in detail. Fourth, a section presents the theory's applications with respect to the various classical empirical regularities, such as between-list strengthening, the list-strength effect, $z$-ROC curves, and response criterion shifting. Fifth, predictions regarding a recently discovered lack of the mirror effect in the context of within-list strength manipulation are presented (e.g., Stretch \& Wixted, 1998a, 1998 b), and an experiment is carried out to evaluate the prediction. For readers who are interested in the analytic solutions of the theory, mathematical derivations of these solutions are presented in the sixth section, and an analysis of the model's optimal performance is conducted. Finally, implications for distributed models of memory and the relations between the variance theory and previous theories of the mirror effect are discussed.

\section{THE VARIANCE THEORY OF THE MIRROR EFFECT}

\section{Overview of the Theory}

In a nutshell, the variance theory proposed here is similar to previous models of the mirror effect, in the sense 
that it also uses vector-feature representations of the items and estimates (via simulations) the response probabilities of old (target) and new (lure) items during a recognition test. However, the variance theory is driven by different conceptual and technical considerations. At the conceptual level, the variance theory sets out to capture the mirror effect mainly in terms of the relations between the study materials and the natural preexperimental context associations the items may have. This is conceptually quite different from all previous theories seeking to explain mirror effects primarily in terms of the individual's decision process. Rather, the approach taken here considers the context in which the individual recognition decision processes takes place. The natural frequencies of events occurring in the individual's day-to-day contexts may be reflected in recognition decision processes, and the individuals may or may not know (or be consciously aware of) these processes. At the technical level, the variance theory also differs from previous theories in a significant way. Instead of directly computing ratios between probabilities, a new way of computing recognition strength is proposed by normalizing the difference between the response probabilities for the target and the lure items with standard deviations of the underlying response distributions.

Specifically, in dealing with the frequency-based mirror effect, a rather plausible key assumption of the variance theory is that high-frequency words are assumed to have appeared in more naturally occurring preexperimental contexts than have the low-frequency words. This assumption is implemented in connectionist network simulations in a rather straightforward way by associating the simulated high-frequency items with more contexts than the low-frequency items during a simulated preexperimental phase. In implementing the theory, items and contexts are represented by two separate arrays (vectors) of binary features, with each feature being represented by a node (or element of the vector), as is shown in Figure 2. A particular item, such as CAR, activates some features at the item layer. A particular context, such as REPAIR SHOP, activates some features at the context layer. Different items and contexts may or may not activate some of the same features. The item and context features are fully interconnected with weights. When an item appears in a particular context and certain features are activated, the weights that reciprocally connect the item features to the context features are adaptively changed according to a specific learning rule, described later. Note that in the implementation, two types of contexts - namely, the preexperimental contexts and the study context (representing internally generated time or list information associated with an item during the actual experimental episode)-are represented in the network, using one common context layer. But these two types of context information are differentiated by two simulation phasesnamely, the preexperimental phase and the actual encoding and testing phase. As will be mathematically outlined later, the standard deviation of the input at the context layer increases when an item is associated with several contexts. Therefore, high-frequency items (associated with more preexperimental contexts) will have larger standard deviations than will low-frequency items in their activation patterns, which are subsequently propagated to the item layer. However, the expected value of the input is equal for high- and low-frequency items.

During the recognition test, an item vector is presented to reinstate the activation of the item features. The features of the study context are reinstated by presenting the network with the context pattern encoded during the study-encoding phase (but not from other preexperimental contex ts that the network was trained with during the preexperimental phase). The degree of recognition strength is determined by first calculating the net inputs to the context and the item nodes. The net input is the signal a node receives from other active nodes connecting to it, and the strength of the signal determines whether the nodes will be activated or not at retrieval. The net input of a given item node is simply computed as the sum of all weights connected to active nodes. Similarly, the net input of a given context node is simply the sum of all weights connected to active nodes and that particular context node. The net inputs, then, denote the retrieved state of activation patterns at the item and context layers. The subset of item and context nodes that have activation levels exceeding a particular activation threshold at retrieval and that were also active during encoding are then used to calculate the recognition strength. Those nodes whose activation does not exceed the threshold or that were inactive during encoding have no influence on recognition strength. For example, assume that the activation threshold is set to 0.5 , so that any node (item or context) that was active during encoding and whose retrieved activation, during testing, exceeded the value of 0.5 would contribute to recognition strength. Imagine that four nodes out of a total of eight exceed the threshold and are equal to $0.75,1.00,1.25$, and 1.50 . The recognition strength of the item is the percentage of above-threshold nodes $(50 \%)$

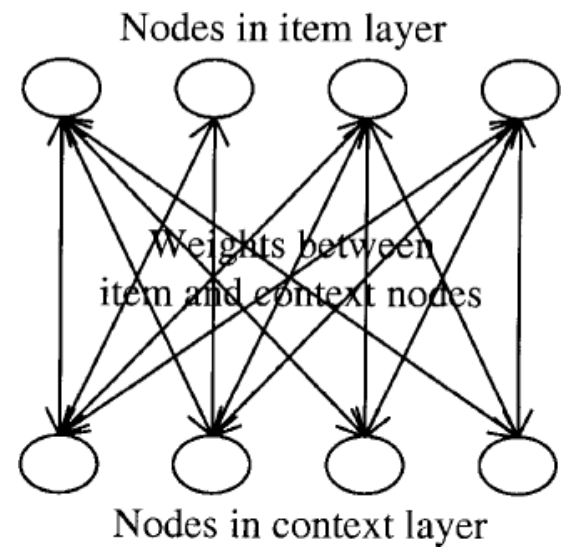

Figure 2. The variance theory. The upper four circles represent nodes in the item layer. The lower four circles represent nodes in the context layer. The arrows between the item and the context layers represent connections. 
minus the expected percentage of above-threshold nodes (e.g., 25\%) divided by the standard deviation of actually observed above-threshold nodes (i.e., by the standard deviation of $0.75,1.00,1.25$, and 1.50 ).

Why is recognition strength determined by this rule, as opposed to, say, just the percentage of above-threshold nodes? As will be shown later, this way of measuring recognition strength (subtracting the expected value and dividing with the standard deviation of the net input) allows the model to perform optimally in terms of discriminability between new and old items when the response bias to the yes response is manipulated. And in this case, the model accounts for why the standard deviation of the response distribution of the easy class is larger than the response distribution of the difficult class. It is plausible to assume that humans have evolved to generally respond more or less optimally and that this is reflected in their performance, as well as in the implementation of the variance theory. Similarly, the activation threshold is set in the model to the value that leads to the highest $d^{\prime}$ (i.e., to optimal performance), which occurs when the activation threshold is between the new and the old distributions. This optimal tuning of the model allows it to account for some rather dramatic results, such as concentering, showing that target and lure distributions from different item classes converge on a strength-of-evidence axis as memory conditions worsen.

Here is a brief example of how the model performs. Consider hypothetical levels of activation generated by high-frequency and low-frequency old (target) and new (lure) items. Because high-frequency words have appeared in a larger number of contexts, they have a larger variance of net input. As such, targets and lures will be relatively more confusable and will generate percentages of activated nodes that are difficult to discriminate. Assume that the standard deviation of the net input is . 10 and the relevant proportions are .25 for high-frequency targets and .15 for high-frequency lures. In contrast, low-frequency words will have occurred in fewer contexts and will be less confusable. Assume that the standard deviation of net inputs is less for low-frequency words-for example, .05-and that the percentage of active nodes is .30 for low-frequency targets and .10 for low-frequency lures. Given these values, what are the recognition strengths for high-frequency and low-frequency targets and lures? If the expected proportion of above-threshold nodes is .20, they are

$$
\begin{aligned}
& \text { Low-frequency lures: }(.10-.20) / .05=-2.0 \\
& \text { High-frequency lures: }(.15-.20) / .10=-0.5 \\
& \text { High-frequency targets: }(.25-.20) / .10=0.5 \\
& \text { Low-frequency targets: }(.30-.20) / .05=2.0 .
\end{aligned}
$$

The model accounts for the various aspects of memory phenomenaby postulating a connectionist neural network model with an implementation and parameter settings that allow it to perform at optimal or near-optimal levels. When the model is optimized, it behaves similarly to how subjects behave, and when it is not optimized, it does not fit the experimental data. This is true not only for the standard mirror effect, but also for exceptions, such as the absence of a mirror effect for within-list strength manipulations (something all other competing formal models fail to do). Furthermore, it predicts key features of the ROCs for new and old items, as well as for highand low-frequency items (something any viable formal model must do).

\section{Presentation of the Variance Theory}

In this section, the details of the variance theory are presented. As will become clearer as the theory is unfolded, the theory is analytical, and the analytical solutions are self-contained solvable (readers who are interested can find the analytical solutions in the sixth section). Although the theory itself does not require a particular computational framework, it can be more easily explained and directly implemented by using a connectionist network. Therefore, the presentation of the theory below is couched within the framework of a Hopfield neural network (Hopfield, 1982, 1984), in order to explicate the theory's underlying mechanisms that generate the mirror effect.

Network architecture. The variance theory may be implemented as a two-layer recurrent distributed neural network (see Figure 2). There are two layers in the representation: one is the item layer, and the other the context layer. Both layers consist of $N$ number of nodes (i.e., $N$ features), although it could also be implemented with an unequal number of context and item nodes. Thus, the total number of nodes in the network is $2 N$. The item and the context layers are fully connected, so that all the nodes in one layer are connected through weights to all the nodes in the other layer. Nodes within a layer are not connected (i.e., no lateral connections).

Stimulus and context representation. Contexts and items are represented as binary activation patterns across the nodes in the context and item layers, respectively. A node is active (or activated) if its corresponding feature is present (i.e., of value 1), and a node is inactive (or not activated) if its corresponding feature is absent (i.e., of value 0 ). There are several reasons for choosing binary representations. For instance, binary representation serves to deblur noisy information at retrieval (Hopfield, 1984). Binary representation also allows for a low proportion of active nodes (spare representation), which is known to improve performance (Okada, 1996). It also introduces nonlinearity, which is necessary for solving some problems in multilayer networks (Rumelhart, Hinton, \& Williams, 1986), and it is perhaps the simplest representation. Furthermore, in the present model, it is shown that it is necessary for capturing characteristics of the $z$-ROC curves that are associated with the mirror effect.

More specifically, the state of activation for the $i$ th node in the item layer at encoding is denoted $\xi_{i}^{d}$, where the superscript $d$ denotes the item layer. The state of activation for the $j$ th node in the context layer at encoding is denoted $\xi_{j}^{c}$, where the superscript $\mathrm{c}$ denotes the context layer. Context patterns and item patterns are generated by randomly setting nodes to an active state (i.e., 
with values of 1) and otherwise to an inactive state (i.e., with values of 0 ). Let $a$ be a parameter that determines the expected probability that a node is active at encoding. This parameter does not change during the simulation and is assumed to be relatively small (for purposes of sparse representation). Note that $a$ is the expected probability of active nodes, whereas the real percentage of active nodes for specific items or contexts varies around $a$.

The encoding-study phase. Encoding occurs by changing the connection weights between the item and the context layers. The weights (or the strengths of the connections) contain information of what has been stored in the network. The weight between item node $i$ and context node $j$ is denoted as $w_{i j}$, and it is initialized to zero. The weight change $\left(\Delta w_{i j}\right)$ is calculated by the learning rule suggested by Hopfield (1982; see also Hertz, Krogh, $\&$ Palmer, 1991, for additional details). This is essentially a Hebbian learning rule that increases connection weights between simultaneously activated nodes. This rule is chosen here because it is more biologically plausible than other rules, such as the delta or the gradientdecent learning rules (e.g., Rumelhart et al., 1986) used in back-propagation networks. However, the variance theory can also be implemented with other learning rules.

According to the Hopfield (1982) learning rule, the weight change is computed as the outer-product between the item and the context vectors of activation patterns, with the parameter $a$ first subtracted from both vectors. This subtraction is mathematically necessary to keep the expected value of the weights at zero. Using the notions for item and context activation defined above, the weight change between these two elements of the item and context vectors can be written as

$$
\Delta w_{i j}=\left(\xi_{i}^{\mathrm{d}}-a\right)\left(\xi_{j}^{\mathrm{c}}-a\right) .
$$

Word frequency as the number of associated contexts at the preexperimental phase. An item may be encoded more or less frequently and, hence, be associated with more or less different preexperimental contexts, depending on how often the item occurs in the subject's environment. In the model, at the preexperimental stage of the simulation, an item's frequency is simulated by the number of times the item is encoded in different contexts. A low-frequency item is encoded less often and is associated with a smaller number of contexts, whereas a highfrequency item is encoded more often and is associated with a larger number of different contexts. For instance, one might form a preexperimental association between $S A A B$ and repair shop after experiencing the rare event of a new expensive SAAB sports car breaking down halfway through a honeymoon trip to the Grand Canyon, with the $\mathrm{SAAB}$ having to be towed to a repair shop somewhere out in the desert! In implementing the variance theory, the relationship between word frequency and preexperimental item-context associations can be simulated straightforwardly. At the preexperimental stage of the simulation, a low-frequency item, SAAB, may be simulated by associating one context item, REPAIR SHOP, with it during encoding. A high-frequency word, CAR, may be simulated by associating three different contexts, REPAIR SHOP, TAXI RIDE, and DRIVING TO WORK, with it during encoding.

The recognition test phase. At recognition, an item is presented to the network, the representation of this item is reinstated as a cue to the item layer, and the representation of the study context (simulating an internally generated context regarding list or time information during the recognition experiment) is reinstated as a cue to the context layer. For example, the representation of the word CAR is reinstated at the item layer. Furthermore, the representation of the study context STUDY LIST is reinstated at the context layer. The subjects must have this information (or cue) in order to recognize an item from the particular study context (and not recognize the item from all the other preexperimental contexts). In the actual experimental setting, this information is usually conveyed to the subjects by the explicit instruction to recognize from the study context (e.g., "Do you recognize the word CAR from the list you just studied?").

At recognition, each node receives a signal (called the net input), which is computed on the basis of other active nodes connecting to it. Item nodes receive net inputs from active context nodes, and context nodes receive net inputs from active item nodes. The net input to a given node is simply the sum of the weights of all other active nodes connected to that node. For example, if item node 1 is connected to four context nodes (1, 2, 3, and 4), where context nodes 1 and 3 are active, the net input to item node 1 is $w_{11}+w_{13}$. Thus, active nodes "send" information to nodes that they are connected to, whereas inactive nodes do not send any information. Put another way, nodes "receive" information or input from active nodes that they are connected to, but not from the inactive nodes.

Specifically, the net input to item node $i$ is calculated by first multiplying the activity of the context node (labeled $j$ ) connected to this node by the weight of the connections between the nodes $i$ and $j$ and then summing over all connected nodes. In vector formalization, the weight matrix operates on the activation vector, and the output is the net input vector. The net inputs to node $i\left(h_{i}^{\mathrm{d}}\right)$ at the item layer depend on the states of activation of nodes in the context layer and the weights connecting these two layers:

$$
h_{i}^{\mathrm{d}}=\sum_{j=1}^{N} w_{i j} \xi_{j}^{\mathrm{c}} .
$$

Following the same principle, a similar function is used to calculate the net inputs to the context node. Specifically, the net inputs to context nodes (labeled $j$ ) depend on all the states of activation of nodes in the item layer and the weights connecting the two layers:

$$
h_{j}^{\mathrm{c}}=\sum_{i=1}^{N} w_{j i} \xi_{i}^{\mathrm{d}} .
$$

By inserting Equation 3 into Equation 4 and summing over the $p$ number of encoded patterns during list learning, it is easy to see that the net input to a node is simply the sum of $N p$ number of terms; for example, the net input to an item node is 


$$
h_{i}^{\mathrm{d}}=\sum^{p} \sum_{j=1}^{N}\left(\xi_{i}^{\mathrm{d}}-a\right)\left(\xi_{j}^{\mathrm{c}}-a\right) \xi_{j}^{\mathrm{c}} .
$$

For $a=.5$, the net inputs are binomially distributed with a certain expected value. Given a certain criterion (i.e., $N p(1-a) a>10)$, a binomial distribution can be approximated with a normal distribution (Feller, 1968). For $a \neq .5$, there are actually four outcomes; however, the same normal approximation can be used. Thus, for reasonably large parameter values of $N p a$, the distribution of net inputs to the nodes can be approximated by a normal distribution.

If the to-be-recognized item has not been encoded with the context (i.e., a new item), the net input is simply the sum of random weights. Because the expected values of all weights are zero, the expected value of the net inputs for new items will also be zero. If the item has been encoded with the context (i.e., an old item), the net input is the sum of those weights connected to that node whose respective context nodes were active at encoding. Owing to the adaptive weight changes during encoding, these weights will have an expected value that is larger than zero if both nodes were in the active state during encoding [i.e., each weight change at encoding is computed as $\left.(1-a)^{2}\right]$ and less than zero if one node was inactive and the other node was active at encoding [i.e., each weight change at encoding was $-a(1-a)$ ]. Of specific importance for the theory is that the variance of the net inputs to the context nodes (from the item nodes) increases with the number of contexts that are associated with the item. Therefore, the variance of the net input is larger for highthan for low-frequency items. Similarly, the variance of the net input to the item nodes (from the context nodes) increases with the number of items associated with one context (i.e., list length). Therefore, given that the context is constant during a list presentation, the variance of the net inputs is larger for a long than for a short list.

Brief summary of optimal performance. Given the strong selection pressure, arguably, humans and animals have evolved to achieve good memory performance. Therefore, it is reasonable to assume that mechanisms for recognition decision have evolved to an optimal or nearoptimal performance. Following this assumption, the parameter values in the model and the implementation of the model are guided by the idea that the model should perform optimally. A detailed discussion about the issue of optimal performance with exact derivations of what is optimal performance in the context of the present model is presented later in the sixth section. Here, I give a brief summary explaining the results from the analysis of optimal performance, without going into the mathematical details (see Figures 9A, 9B, 9C, and 9D).

The model's performance is optimal if the percentage of nodes active at encoding (a) is low (see Figure 9A). For a low $a$, it is optimal to base the recognition decision on nodes that were active at encoding and to ignore nodes that were inactive during encoding (see Figure 9A). Also, for a low $a$, it is optimal to place the activation threshold of the nodes between the expected values of the new and the old net inputs (see Figure 9B). Finally, it is optimal to normalize the recognition strength with the standard deviation of the net input (see Figures 9C and 9D).

For a low percentage of active nodes, it is optimal to base the recognition decision on nodes that were active at encoding (or nodes active in the cue pattern) and to ignore nodes that were inactive at encoding. At recognition, the state of activation of a node may be either active or inactive. Therefore, the nodes that are active in the cue pattern and have a net input above a certain activation threshold are activated at recognition; otherwise, the nodes are inactivated. Let $\zeta_{i}^{\mathrm{d}}$ denote the state of activation at recognition for item node $i$. An item node is activated at recognition $\left(\zeta_{i}^{\mathrm{d}}=1\right)$ if it was active in the cue pattern $\left(\xi_{i}^{\mathrm{d}}=1\right)$ and the net input is above the activation threshold $\left(h_{i}^{\mathrm{d}}>T\right)$; otherwise, it is inactivated $\left(\zeta_{i}^{\mathrm{d}}=0\right)$ :

$$
\zeta_{i}^{\mathrm{d}}=1 \text {, if } \xi_{i}^{\mathrm{d}}=1 \text { and } h_{i}^{\mathrm{d}}>T \text {; otherwise, } \zeta_{i}^{\mathrm{d}}=0 .
$$

Similarly, let $\zeta_{j}^{\mathrm{c}}$ denote the state of activation at recognition for context node $j$ :

$$
\zeta_{j}^{c}=1 \text {, if } \xi_{j}^{c}=1 \text { and } h_{j}^{c}>T \text {; otherwise, } \zeta_{j}^{c}=0 .
$$

This way of activating nodes at retrieval differs from how nodes are activated in a standard Hopfield (1982) network, where the activation threshold is zero and a node is activated if the net input is above zero (independently of the state of activation in the cue pattern). The way of activating patterns in a Hopfield network is more likely to produce a retrieved pattern that matches the encoded pattern of activation (e.g., the expected value of active nodes at retrieval will be the same as the expected value of active nodes at encoding). However, as will be discussed later, the way suggested to activate the nodes here yields better performance in terms of discrimination between a target item and a distractor item.

As is shown in Figure 9B, performance is optimal when the activation threshold is set approximately between the new and the old net inputs. The activation threshold $(T)$ is set to the expected values of the net inputs of nodes active during encoding $\left(\xi_{i}^{\mathrm{d}}=1, \xi_{j}^{\mathrm{c}}=1\right)$ for old and new items. The averaging is computed over all nodes $(2 N)$ and over all new and old patterns $(p)$ in the recognition condition. If half of the items are new and half of items are old, the activation threshold is

$$
T=\frac{1}{2 a P N} \sum^{P}\left[\sum_{i=1}^{N} \xi_{i}^{\mathrm{d}} h_{i}^{\mathrm{d}}+\sum_{j=1}^{N} \xi_{j}^{\mathrm{c}} h_{j}^{\mathrm{c}}\right] .
$$

As was discussed above, the expected net input of new (lure) items is zero. Therefore, the activation threshold is simply half the expected net input for nodes encoded in the active state $\left[T=\mu_{h}(\mathrm{O}) / 2\right.$, where $\mu_{h}(\mathrm{O})$ is the expected value of the net input to nodes encoded in the active state].

It is easy to see that the expected percentage of old and new active nodes at recognition is one half of the percentage of active nodes at encoding $(a / 2)$. That is, the activation threshold divides the old and new distribution in 
the middle. Old items will have a higher expected percentage active, and new items will have a lower expected percentage active. The activation threshold is constant during recognition in one condition. However, it must vary between conditions, depending on the net inputs, to yield optimal performance.

The percentage of nodes active at recognition is counted:

$$
P_{c}=\frac{1}{2 N}\left(\sum_{i=1}^{N} \zeta_{i}^{\mathrm{d}}+\sum_{j=1}^{N} \zeta_{j}^{\mathrm{c}}\right) .
$$

As is shown in Figure 9C, the performance is near-optimal if the recognition decision is based on the number of nodes active at recognition, normalized by the standard deviation of the net inputs across the active features of this item $\left(\sigma_{h}^{\prime}\right)$. Thus, this standard deviation is calculated over all the nodes active at encoding (i.e., $\xi_{i}^{\mathrm{d}}=1$ and $\xi_{i}^{\mathrm{c}}=1$; nodes inactive at encoding are not used when calculating the standard deviation because, for low levels of $a$, these items carry little to no information of the item). The recognition strength $(S)$ for an item is calculated by subtracting the expected percentage of nodes active at recognition $(a / 2)^{1}$ from the real percentage of nodes active at recognition $\left(P_{c}\right)$ and dividing by the standard deviation of the net inputs of the item $\left(\sigma_{h}^{\prime}\right)$ :

$$
S=\frac{P_{c}-\frac{a}{2}}{\sigma_{h}^{\prime}}
$$

The subtraction of the expected percentage of nodes active at recognition makes the expected value of the recognition strength $(S)$ zero. This subtraction is necessary for the normalization to work properly. The subtraction moves the recognition strength distributions symmetrically, so that the old and the new distributions move at the same rate for a given standard deviation of the net input (without the subtraction, the old recognition strength distribution would be more affected than the new distribution). Thus, the recognition strength is determined by the difference between two probabilities (the percentage of active nodes that varies and the expected percentage of active nodes that is constant) divided by the standard deviations of the net input. A yes response $(Y)$ is given if the recognition strength $(S)$ is above the recognition criterion $(C)$. An unbiased decision has a recognition criterion of zero:

$$
Y=S>C \text {. }
$$

An issue that may be raised is whether it is sensible to base recognition strength on two quite different sourcesnamely, the percentage of active nodes and the variability of the net input. The immediate answer is that if it reasonable to optimize performance, it is also sensible to measure recognition strength this way. Another perspective is to note that unbiased responses can be made only on the percentage of active nodes - that is, a yes response occurs if the percentage of active nodes is larger than the expected percentage of active nodes $\left(P_{\mathrm{c}}>a / 2\right)$ and the variability of the net input can be ignored. Thus, "nor- mally," subjects base their unbiased decisions on the percentage of active nodes, and the variability of active nodes only becomes relevant when subjects are biased. From this perspective, the percentage of active nodes is used for unbiased responses, and the variability of the net input becomes relevant for confidence judgments. Therefore, by combining both the percentage of active nodes and the variability of the net input, the measure of recognition strength proposed here will also reflect the confidence judgment.

\section{An Example With Step-by-Step Computations}

To clarify the computational details involved in the variance theory, a step-by-step example is given here. For tractability, a small network is used consisting of four item nodes and four context nodes (see Figure 2). The actual simulations to be reported later involved a larger network architecture with 30 nodes at each layer. The percentage of nodes active at encoding (denoted by parameter $a$ ) is set to .50 . Let item $\mathrm{BN}$ be represented as $\{1,1,0,0\}$ and written as the state of activation of the four nodes $\left\{\xi_{1}^{\mathrm{d}}, \xi_{2}^{\mathrm{d}}, \xi_{3}^{\mathrm{d}}, \xi_{4}^{\mathrm{d}}\right\}$. Similarly, let $\{0,0,1,1\}$ represent item $\mathrm{BO},\{1,0,1,0\}$ represent item $\mathrm{AO}$, and $\{0,1,0,1\}$ represent item $A N$. Let context $C_{B N}$ be represented as $\{1,1,0,0\}$, or the state of activation of the four nodes $\left\{\xi_{1}^{\mathrm{c}}\right.$, $\left.\xi_{2}^{\mathrm{c}}, \xi_{3}^{\mathrm{c}}, \xi_{4}^{\mathrm{c}}\right\}$. Similarly, $\{0,0,1,1\}$ represents context $\mathrm{C}_{\mathrm{BO}}$ and $\{0,1,0,1\}$ represents experimental study context $\mathrm{C}_{\mathrm{Exp}}$.

Item $\mathrm{BN}$ is a high-frequency new word. For simplicity, it is here encoded only once with context $\mathrm{C}_{\mathrm{BN}}$ in the preexperimental phase (in the simulations below, highfrequency words are preexperimentally associated with three contexts). The 16 weights between the four item nodes and the four context nodes are changed according to the learning rule, where the probability that a node is active at encoding is determined by the parameter $a=$ .5. For example, the weight change between item node 1 and context node 1 is $w_{11}=\left[\xi_{1}^{\mathrm{d}}(\mathrm{BN})-a\right]\left[\xi_{1}^{\mathrm{d}}\left(\mathrm{C}_{\mathrm{BN}}\right)-a\right]=$ $(1-.5)(1-.5)=1 / 4$, where $\mathrm{BN}$ is item $\mathrm{BN}$ and the $\mathrm{C}_{\mathrm{BN}}$ represents context $\mathrm{C}_{\mathrm{BN}}$. Similarly, item $\mathrm{BO}$ is another high-frequency word that, before the experimental phase, is encoded once with context $\mathrm{C}_{\mathrm{BO}}$. Items $\mathrm{AO}$ and $\mathrm{AN}$ are low-frequency old and new words, and they are not encoded at the preexperimental phase.

In the experimental phase, item $\mathrm{AO}$ is encoded with the experimental context $\mathrm{C}_{\text {Exp. }}$. Finally, item $\mathrm{BO}$ is encoded with the same experimental context $\mathrm{C}_{\text {Exp }}$. For example, the weight $w_{11}$ is now equal to

$$
\begin{aligned}
& {\left[\xi_{1}^{\mathrm{d}}(\mathrm{BN})-a\right)\left(\xi_{1}^{\mathrm{c}}\left(\mathrm{C}_{\mathrm{BN}}\right)-a\right]+\left[\xi_{1}^{\mathrm{d}}(\mathrm{BO})-a\right]} \\
& {\left[\xi_{1}^{\mathrm{c}}\left(\mathrm{C}_{\mathrm{BO}}\right)-a\right]+\left[\xi_{1}^{\mathrm{d}}(\mathrm{BO})-a\right]\left[\xi_{1}^{\mathrm{c}}\left(\mathrm{C}_{\mathrm{E}}\right)-a\right]} \\
& +\left[\xi_{1}^{\mathrm{d}}(\mathrm{AO})-a\right]\left[\xi_{1}^{\mathrm{c}}\left(\mathrm{C}_{\mathrm{E}}\right)-a\right]=(1-.5)(1-.5) \\
& +(0-.5)(0-.5)+(0-.5)(0-.5) \\
& +(1-.5)(0-.5)=1 / 4+1 / 4+1 / 4-1 / 4=1 / 2 .
\end{aligned}
$$

After encoding, the full weight matrix is $\{\{.5,1,-1,-.5\}$, $\{.5,0,0,-.5\},\{-.5,0,0, .5\},\{-.5,-1,1, .5\}\}$, corresponding to the weights $\left\{\left\{w_{11}, w_{12}, \ldots w_{44}\right\}\right\}$, respectively. 
At recognition, the state of activation of the old lowfrequency item $\mathrm{AO}$ is reinstated as a cue to the item layer, and the state of activation of the encoding context at the experimental phase $\mathrm{C}_{\operatorname{Exp}}$ is reinstated as a cue to the context layer. The net inputs are calculated. For example, the net input to context node 1 is

$$
h_{1}^{\mathrm{c}}=\sum_{i=1}^{4} w_{1 i} \xi_{i}^{\mathrm{d}}=.5 * 1+1 * 0-1 * 1-.5 * 0=-5 .
$$

The net input to the item nodes is $\{1,0,2,1\}$, and that to the context nodes is $\{-.5, .5,-.5, .5\}$. It can be seen that the expected net inputs for randomly created new items in this network is 0 and that the expected net inputs for old items encoded in the active state is 0.5 . Therefore the activation threshold is set to the average of these valuesnamely, $T=0.25$. Nodes that have a net input above the activation threshold and that are active in the cue pattern are activated at recognition. Thus, the state of activation at recognition for the item nodes is $\{1,0,1,0\}$, and that for the context nodes is $\{0,1,0,1\}$ (which is identical to the cue patterns). The percentage of active nodes is counted $P_{c}(\mathrm{AO})=4 / 8=.5$. For an unbiased response $(C=0)$, this will yield a yes response, because this percentage is larger than the expected percentage of active nodes at recognition $(a / 2=.25)$. The standard deviation of the net input for nodes active at encoding is 0.71 (i.e., the standard deviation of $\{1,2,-.5, .5\}$, corresponding to nodes $1,3,6$, and 8 ). The recognition strength is calculated by subtracting the expected percentage of active nodes at recognition from the percentage of active nodes for the to-be-recognized item and dividing by the standard deviation of the net input for active nodes $\left[S=\left(P_{c}-\right.\right.$ $\left.a / 2) / \sigma_{h}^{\prime}=(.5-.25) / .71=0.35\right]$.

The recognition of the three items $\mathrm{BO}, \mathrm{AN}$, and $\mathrm{BN}$ are done in the same way. The results for the four items $\mathrm{AN}, \mathrm{BN}, \mathrm{BO}$, and $\mathrm{AO}$ are the net inputs $(1,0,2,1, .5,1.5$, $.5,-.5,-1.5)$, where the first four numbers represent item nodes, and the last four, context nodes $(1,0,2,1$, $1.5, .5,-.5,-1.5),(1,0,2,1,-1.5,-.5, .5,1),(1,0,2$, $1,-.5, .5,-.5, .5)$, the states of activation at recognition $(0,0,0,1,0,0,0,0),(1,0,0,0,0,1,0,0),(0,0,1,1,0$, $0,0,1),(1,0,1,0,0,1,0,1)$, the numbers of nodes active $1,2,3,4$, the standard deviations of the net inputs $0.71,1.08,1.08,0.71$, the recognition strengths $-.17,0$, $0.11,0.35$, and the unbiased responses no, no, yes, yes, respectively (an unbiased yes response is made when $S>C$, where $C=0$ for unbiased responses). Thus, the subject responds correctly for all items, the recognition strengths are ordered according to the mirror effect, and the variance of the net input is larger for the high- than for the low-frequency words.

\section{SIMULATIONS OF THE FREQUENCY-BASED MIRROR EFFECT}

In this section, the variance theory of the mirror effect is simulated in a connectionist framework that is consistent with a general connectionist theory of memory called TECO (Sikström, 1996a, 1996b). TECO has been used to account for a variety of memory phenomena involving recognition and recall—for example, successive testing of recall and recognition (Sikström, 1996b) and forgetting curves (Sikström, 1999). An extensive description of TECO is beyond the scope of this paper. Interested readers are referred to previous articles describing the model for details.

\section{Procedure}

The simulation started with initializing the weights to zero. Then, 12 items were generated by randomly setting the nodes to an active state with a probability of $a$. A preexperimental phase then followed to generate the frequency associated with the items. In this preexperimental phase, half of the items (i.e., 6) were encoded three times, each time in a different context. These items simulated the high-frequency words. The remaining 6 items were not encoded before the experimental phase, and they simulated the low-frequency words.

At the experimental phase, one study-encoding context was generated, using the same procedure as the generation of the contexts in the preexperimental phase. Given that in a standard recognition experiment, all studied items would be encoded in the same list, in the simulations the items were thus encoded once with the same study context. Three of the high-frequency items were encoded, and three of the low-frequency items were encoded. The other three high- and three low-frequency items were not encoded during the experimental phase, and they simulated the new items. Each encoding was simulated by first activating the nodes in the item and context layers. The weights were then changed according to the learning rule (Equation 3).

At the recognition test, the patterns of activation of the 12 items and the study context were reinstated to the network. The net inputs were calculated for each node, and the recognition strength was calculated from all the nodes in the network. The somewhat unrealistic assumption that no learning, or changes of weights, occurred during testing was adopted. However, this is a standard assumption often used in other simulations of recognition memory, cued recall, or categorization (e.g., Kruschke, 1992; Lewandowsky, 1991; Li \& Lindenberger, 1999; Li, Lindenberger, \& Frensch, 2000). One thousand five hundred simulation runs with different random item and context patterns were carried out. The results reported later are based on the average across these runs.

\section{Parameters}

The number of high-frequency patterns was six (each encoded three times preexperimentally and three of them encoded once experimentally to simulate the old items), and the number of low-frequency patterns was six (three of them encoded experimentally once to simulate the old items). The following parameter settings were used: The number of nodes in each layer $(N)$ was 30 (the total number of nodes was $2 N=60$ ), and the percentage of nodes 

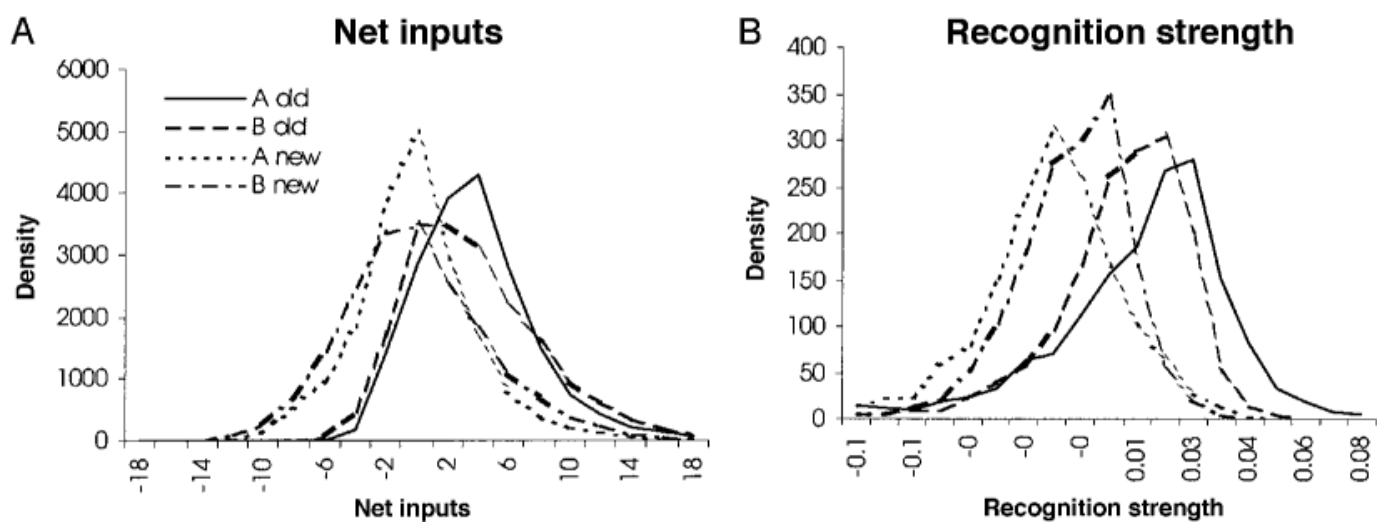

Figure 3. (A) Simulations of net inputs. The vertical axis shows the simulated density of the net inputs. (B) Simulations of the mirror effect. The vertical axis shows the simulated density of the recognition strength.

active at encoding (a) was .20. Initially, all the weights were set to zero. The recognition criterion $(C)$ was set to 0 .

\section{Results}

Figure 3A shows the results in terms of the density of the net inputs to an active node. The expected values of the net inputs are approximately equal for new high- and new low-frequency items $\left[\mu_{h}(\mathrm{AN})=0.0, \mu_{h}(\mathrm{BN})=0.0\right]$ and for old low- and old high-frequency items $\left[\mu_{h}(\mathrm{AN})=\right.$ $\left.3.8, \mu_{h}(\mathrm{BN})=3.8\right]$. The high-frequency items have a larger variance of the net inputs $\left[\sigma_{h}(\mathrm{BN})=4.9, \sigma_{h}(\mathrm{BO})=\right.$ $4.8]$ than do the low-frequency items $\left[\sigma_{h}(\mathrm{AN})=4.1\right.$, $\left.\sigma_{h}(\mathrm{AO})=4.0\right]$. The variances of the old and the new distributions are approximately equal.

Figure 3B shows the density of the recognition strength. The result shows the mirror effect where the hit rate probability is larger for low- than for high-frequency items and the false alarm rate is lower for low- than for highfrequency items $[P(\mathrm{AN})=.18, P(\mathrm{BN})=.25, P(\mathrm{BO})=$ $.64, P(\mathrm{AO})=.71]$. The standard deviation for the recognition strength is larger for old than for new words and larger for the low-frequency words than for the highfrequency words $\left[\sigma_{s}(\mathrm{AN})=0.29, \sigma_{s}(\mathrm{AN})=0.19, \sigma_{s}(\mathrm{AN})\right.$ $\left.=0.23, \sigma_{s}(\mathrm{AN})=0.31\right]$. These findings agree with the empirical data and the predictions of the attentionlikelihood model (Glanzer et al., 1993). Thus, the simulation shows that the variance theory can account for the frequency-based mirror effect and the associated ROC curves.

\section{EXPLICATIONS OF THE MECHANISMS}

In this section, three essential mechanisms of the variance theory that account for the mirror effect are discussed in detail. The involved mechanisms are (1) the variance of the net input, (2) the expected value of the net input, and (3) the way in which the recognition strength is calculated by counting the number of nodes so that the performance is optimal.

\section{Overview}

The first mechanism is that items from the difficult class (i.e., high-frequency items, in this case) have a higher variance of the net inputs than do items from the easy class (i.e., low-frequency items), but the variance is independent of whether the items are old or new. The mechanism is illustrated in Figure 4A (noncumulative). It should also be underscored that the difference in variance as a function of class membership is not a primitive of the theory that the theorist manipulates. It is the natural consequence of the rather plausible assumption that highfrequency items appear more often and are associated with a great variety of different contexts than are lowfrequency words. The second mechanism is that old items have a higher expected net input to nodes encoded in the active state than do new items (but do not depend on the class of the items). The mechanism is illustrated in Figure 4B (cumulative). The third mechanism is the way recognition strength is measured by counting the active nodes so that recognition performance (e.g., $\left.d^{\prime}\right)$ is optimal or near-optimal. An extended analysis of optimality is presented at the end of the paper; however, the results in Figures 9A-9D can summarize the main points here. The results from this analysis imply that the decision must be based on the nodes active at encoding (see Figure 9A), the percentage of active nodes must be low (see Figure 9A), the activation threshold needs to be between the expected net inputs of the new and old items (see Figure 9B), and the recognition strength is normalized by the standard deviation of the net inputs of the item (see Figures 9C and 9D). The density of the percentage of active nodes is illustrated in Figure $4 \mathrm{C}$, and the normalized percentage of active nodes is illustrated in Figure 4D. Here, it is shown how these mechanisms predict the mirror effect. Below these three sets of mechanisms are explained in more detail.

\section{Variance of the Net Input}

The first, and perhaps the most important, mechanism is that the difficult class has a larger variability in the net 

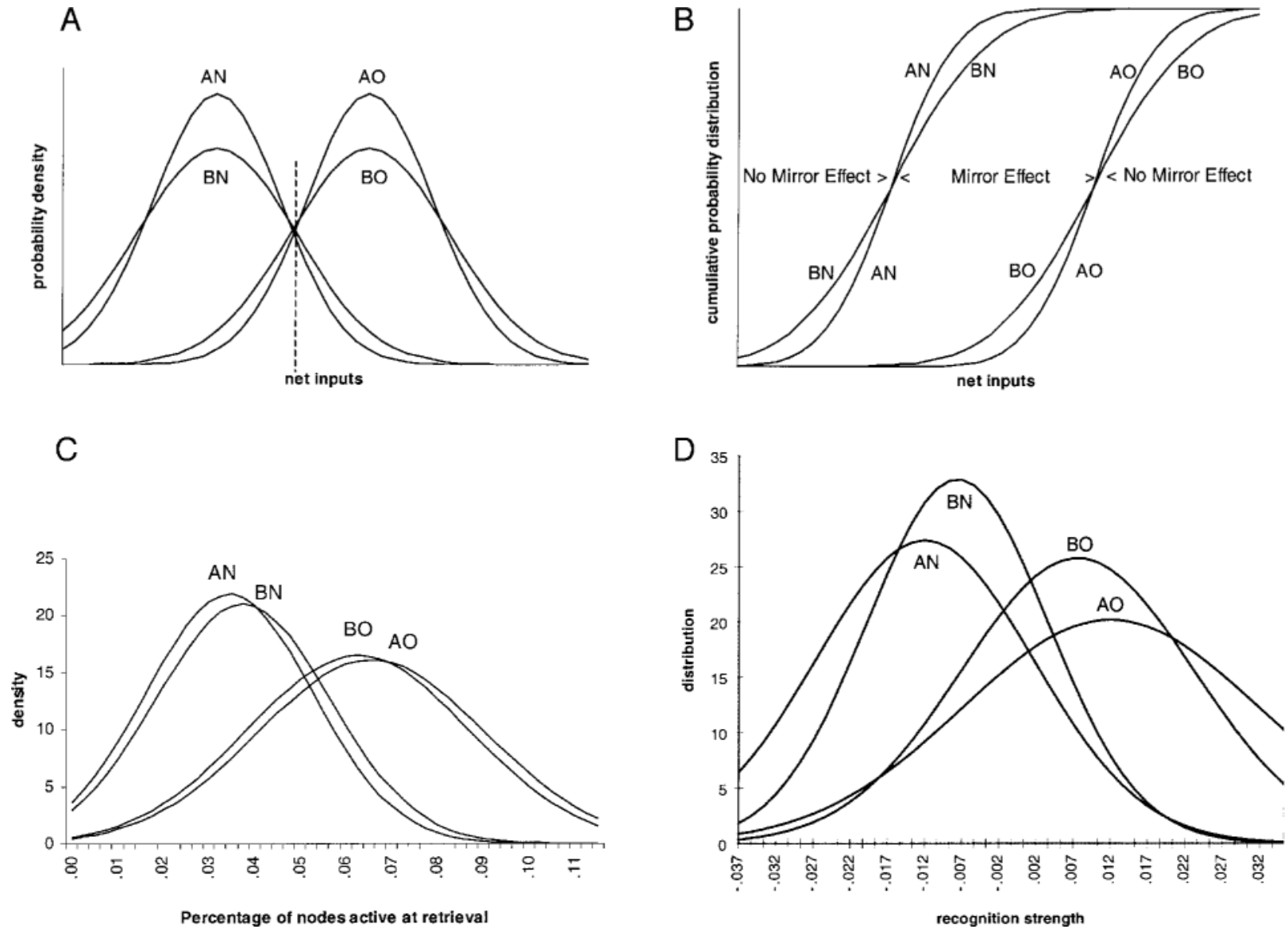

Figure 4. (A) The probability density of the net inputs in the variance theory. The horizontal axis shows the net inputs, and the vertical axis the probability density of the net inputs. The dotted vertical line is the activation threshold. (B) The cumulative probability distributions of the net inputs in the variance theory. The horizontal axis shows the net inputs, and the vertical axis the cumulative probability distributions of the net inputs. (C) The density of percentage nodes active at recognition in the variance theory. The horizontal axis shows the percentage of nodes active at recognition, and the vertical axis the density. (D) The density of recognition strength in the variance theory. The horizontal axis shows the recognition strength, and the vertical axis the density of the recognition strength, using standard parameter values.

inputs than does the easy class. As will be discussed later, this is shared with other theories, such as the subjectivelikelihood account (McClelland \& Chappell, 1998) and REM (Shiffrin \& Steyvers, 1997). However, a unique aspect of the variance theory is that it is a necessary outcome of simply encoding high-frequency items more times than low-frequency items. In the subjective-likelihoodaccount, a larger variability for high-than for low-frequency words is an assumption that is controlled by a free parameter $\left(p_{0}\right)$, to reflect that high-frequency words have more definitions than do low-frequency words. The variance theory needs no such assumptions and no additional free parameters to control the variability. Whereas the subjective-likelihood account tries to capture word frequency with a free parameter, in the variance theory the word frequency effect is simulated via the number of contexts associated with an item.

Owing to how the variance theory implements the relations between contexts and items, the variance of the net inputs increases with the frequency with which an item is encoded in different contexts. Intuitively, this occurs because a high-frequency item activates several different contexts, causing the representations of many competing contexts to be activated simultaneously. Low-frequency items are associated with fewer contexts than are highfrequency items. This causes the representations of fewer contexts to be activated simultaneously. Thus, lowfrequency items have less variability in the net inputs than do high-frequency items.

At recognition, an item produces a net input in the context layer that is a mixture of the net inputs from the study context that the network is instructed to retrieve from and from several uncorrelated preexperimental contexts that were associated with the item during the preexperimental phase. The study context that the network is instructed to retrieve from contributes to the correct active state. For example, by adding +1 to the net input to a node (which is the expected net input for a node encoded 
in the active state when $N=8$ and $a=.5$; see Equation A1 in the Appendix), thus increasing the expected net input. The preexperimental contexts, on the other hand, randomly add to or subtract from the net input. For example, a preexperimental context could add +1 to the net input if the node was in an active state, or it could add -1 to the net input if the particular preexperimental context was encoded in an inactive state (which is the expected net input for a node encoded in the inactive state when $N=8$ and $a=.5$; see Equation 3, 4, or A1). Note that the net input can be negative, whereas the state of activation cannot. If the representations of these preexperimental contexts are uncorrelated, the number of associated preexperimental contexts will not influence the expected net input. That is, the expected value of the negative and positive contributions will cancel out (e.g., for $N=8$ and $a=1$, the contributions to the net input is +1 with a probability of $50 \%$ and -1 with a probability of $50 \%$, yielding an expected value of 0 ). However, the variability of the net inputs increases when more contexts are added. In this example, the variance is increased by $1^{2}$ for each added context (i.e., the variance increases by each contribution raised to the power of two). Encoding an item also increases the variability of the net input for all other items encoded in the network. However, the increase in variability for the items uncorrelated with the encoded item is much smaller, because the contribution from each node is independent. (E.g., for $N=8$ and $a=.5$, the contribution from each node is either $+1 / 4$ or $-1 / 4$ [see Equation 3], each yielding an increase in variability of $1 / 4^{2}=1 / 16$. An expected value of $a N$ nodes contribute, so the total increase in variability is $4 * 1 / 16=1 / 4$.)

A mathematical analysis of how the variability of the net inputs in the context nodes increases as a function of the frequency with which the item has been encoded in different contexts is presented in the Appendix. This analysis shows that the variance of the net inputs in context nodes increases linearly with how many times a given item is encoded within different contexts. The variability of the net inputs for nonwords may be a special case, discussed at the end of this paper.

In the same way as the variability of context nodes depends on the item's frequency, the variability of the item nodes depends on the frequency of the context. That is, the variability of the net input to the item nodes increases with how many times one context is associated with different items. Given that the context is constant during the presentation of a study list, the variability of the net inputs to the item nodes will increase with list length.

\section{Expected Net Input}

The second mechanism in the variance theory is that the expected net inputs to the easy and difficult classes of stimuli are equal, given that the encoding conditions during the experimental phase of the two classes are equal. Note that this is in stark contrast to the attentionlikelihood theory, which assumes that more attention (corresponding to more net input) is given to the easy class than to the difficult class. Experimentally, the equal- ity of the net inputs simply means that different classes of stimuli are given the exact same conditions for encoding and retrieval in a recognition memory study. The net input to a node encoded in the active state increases during encoding, whereas the net input to a node encoded in the inactive state decreases during encoding. Only nodes encoded in the active state are used during retrieval, so here we are only interested in the increase in net input that occurs for nodes encoded in the active state. Strengthening of the weights during encoding increases the expected net input. The degree of increase in expected net input is influenced by strength-based variables, such as study time, repetition, levels of processing, and so forth. For example, the simulations can be set up so that a study time of $1 \mathrm{sec}$ strengthens the weights less, leading to lesser increases in the net input, than does a longer period of study time-for example, $2 \mathrm{sec}$ of encoding time. Because the study context is unique to the learning episode, preexperimental encoding in other learning contexts will not affect the expected net input, but they do affect the variability of the net input, as was demonstrated above. The item-study-context associations are learned approximately equally well for old high- and old low-frequency items. For example, the expected net input for CAR (a high-frequency word as a difficult class item) is equal to the expected net input for ARCTIC (a low-frequency word as an easy class item). Generally, the expected net input does not depend on the class of the item, because the expected net input is influenced by the study and the testing experimental conditions similarly across stimulus classes in a recognition memory experiment. Therefore, the expected net input for a new difficult item is equal to the expected net input for a new easy class item.

The probability density functions of the net inputs for nodes in the active states are plotted in Figure 4A. Old nodes in the inactive state have a negative expected value of the net input and are not plotted. New nodes in the inactive state have the same density as nodes in the active state. The cumulative probability distributions of the net inputs for nodes in the active state are plotted in Figure 4B. Figure 4A shows the first mechanism-namely, that the standard deviation of net inputs for the easy class items $\left[\sigma_{h}(\mathrm{~A})\right]$ is larger than the standard deviation of net inputs for the difficult class items $\left[\sigma_{h}(\mathrm{~B})\right]$. The second mechanism is shown in the figures in that the expected net input of an easy class new item $\left[\mu_{h}(\mathrm{AN})\right]$ is equal to the expected net input of a difficult class new item $\left[\mu_{h}(\mathrm{BN})\right]$. Furthermore, at encoding, the expected net inputs of activated nodes are increased equally or approximately equally for the easy and the difficult classes of items. This is shown in Figure 4A. The expected net input for the old easy class items $\left[\mu_{h}(\mathrm{AO})\right]$ is equal to the expected net input for the difficult class items $\left[\mu_{h}(\mathrm{BO})\right]$.

\section{Recognition Strength}

The variance theory suggests that the recognition decision needs to be based on counting the number of active nodes in such a way that the performance is optimal or near-optimal. If the net input is above the activation 
threshold $(T)$ and the node was active at encoding, the node is activated at recognition. Otherwise, the node is inactivated. The distributions of active nodes are plotted in Figure 4C.

A closer inspection of Figures $4 \mathrm{~A}$ and $4 \mathrm{~B}$ reveals that these densities or distributions predict the correct order of the mirror effect, given that the activation threshold is larger than the expected net inputs of the new items and less than the expected net inputs of the old items. Thus, the variance theory has the nice property of accounting for the mirror effect across a large range of the parameter values for the activation threshold. Thus, $P(\mathrm{AN})<P(\mathrm{BN})<$ $P(\mathrm{BO})<P(\mathrm{AO})$ for $\mu_{h}(\mathrm{AN})=\mu_{h}(\mathrm{BN})<T<\mu_{h}(\mathrm{AO})=$ $\mu_{h}(\mathrm{BO})$. The variance theory predicts a material-based mirror effect, because the variability of the net inputs is different for the easy and the difficult class items. The expected strengths of the net inputs are equal. The variability is lower for easy class items, thus making the probability of false alarms (or the probability of active nodes) lower for the easy than for the difficult class items when the activation threshold is larger than the expected value of the new items. Similarly, the hit rate (or the probability of active nodes) for easy class items is higher than the hit rate for difficult class items when the activation threshold is less than the expected value of the old items.

The activation threshold is set to be between the expected value of the new and the old net inputs so that the performance is optimal. Therefore, the activation threshold is set to the average of the expected net inputs of the old and the new distributions for difficult and easy class items, respectively:

$$
\begin{aligned}
T & =\frac{1}{4}\left[\mu_{h}(\mathrm{AN})+\mu_{h}(\mathrm{BN})+\mu_{h}(\mathrm{BO})+\mu_{h}(\mathrm{AO})\right] \\
& =\frac{1}{4}\left[\mu_{h}(\mathrm{BO})+\mu_{h}(\mathrm{AO})\right]=\frac{1}{2} \mu_{h}(\mathrm{O}) .
\end{aligned}
$$

Thus, in the variance model, the activation threshold is fixed for recognition in one condition, although it may vary between different recognition conditions to optimize the performance. The variance theory accounts for the strength-based mirror effect that occurs between lists or conditions with a shift in the activation threshold necessary for keeping the performance at an optimal level. As will be discussed later, this is true also when performance is measured by $d^{\prime}$, and it is independent of the placement of the recognition criterion. Simply put, the model adopts the activation threshold on the basis of the overall difficulty of the test in order to maximize the performance.

In practice, subjects may initially make a preliminary estimate of the activation threshold, which may be adjusted as more information about the expected value of the distributions is gathered. The theory does not show a mirror effect if the activation threshold is lower than the expected value of the new items or larger than the expected value of the old items. Thus, setting the activation threshold, as was suggested above, is an important mechanism in the model. The activation threshold should not be confused with the subject's recognition criterion.

Figure $4 \mathrm{C}$ shows the density of the probability that a node is active at recognition when the activation threshold is set as defined above. Note how the mean and standard deviations of the distributions of the net input (Figure 4A) change when the percentage of nodes are calculated (Figure 4C). The expected probabilities of active nodes are arranged according to the mirror effect $\left[P_{c}(\mathrm{AN})<\right.$ $\left.P_{c}(\mathrm{BN})<P_{c}(\mathrm{BO})<P_{c}(\mathrm{AO})\right]$, whereas the expected values of the net inputs are not $\left[\mu_{h}(\mathrm{AN})=\mu_{h}(\mathrm{BN})<\mu_{h}(\mathrm{BO})=\right.$ $\left.\mu_{h}(\mathrm{AO})\right]$. Furthermore, the standard deviation of the percentage of active nodes for old items is larger than that for new items $\left[\sigma_{p}(\mathrm{O})>\sigma_{p}(\mathrm{~N})\right]$, whereas the standard deviations of the net inputs are equal for old and new items $\left[\sigma_{h}(\mathrm{~N})=\sigma_{h}(\mathrm{O})\right]$.

The standard deviation of the recognition strength is smaller for the new distributions than for the old distributions because there are fewer nodes active in the new distributions. The standard deviation of the percentage of active nodes at retrieval as a function of the expected probability of nodes active at retrieval is plotted in Figure 5. Obviously, the standard deviation of the percentage of active nodes is zero when the probability of active nodes is zero. This standard deviation increases as the probability of active nodes increases. For small probabilities of active nodes, the variance of active nodes $\left(\sigma_{p}\right)$ is approximately proportional to the percentage of active nodes $\left[\sigma_{p}=P_{c}\left(1-P_{c}\right) / N \approx P_{c} / N \propto P_{c}\right]$. The percentage of active nodes is, of course, larger for old than for new items. Thus, the variance theory predicts that the standard deviation of the percentage of active nodes $\left(\sigma_{p}\right)$ is smaller for new than for old items $\left[\sigma_{p}(\mathrm{AN})<\sigma_{p}(\mathrm{BN})<\sigma_{p}(\mathrm{BO})<\right.$ $\left.\sigma_{p}(\mathrm{AO})\right]$, whereas the standard deviation of the net input is not $\left[\sigma_{h}(\mathrm{AN})=\sigma_{h}(\mathrm{AO})<\sigma_{h}(\mathrm{BN})=\sigma_{h}(\mathrm{BO})\right]$. The essential mechanism that makes these changes in the means and standard deviations is the nonlinearity introduced by the counting of the number of active nodes. Without this nonlinearity, these changes would not occur, and the model would not show appropriate ROC curves for old and new items.

Note that the standard deviation of active nodes decreases for probabilities larger than one half (see Figure 5; the standard deviation is, of course, zero when the probability of active nodes is one; see the mathematical analysis below). However, the probability of active nodes cannot exceed one half, because the activation threshold is set so that half of the nodes active during encoding are active at recognition. The probability of active nodes is typically set to a small value in the model because it improves the performance.

To optimize the performance, subjects base their recognition decision on the number of active nodes normalized by the standard deviation of the net inputs for the item. The normalization makes the judgments more conservative for difficult items. This plays an important role for confidence judgments when the responses are biased, but plays no role for unbiased responses. 


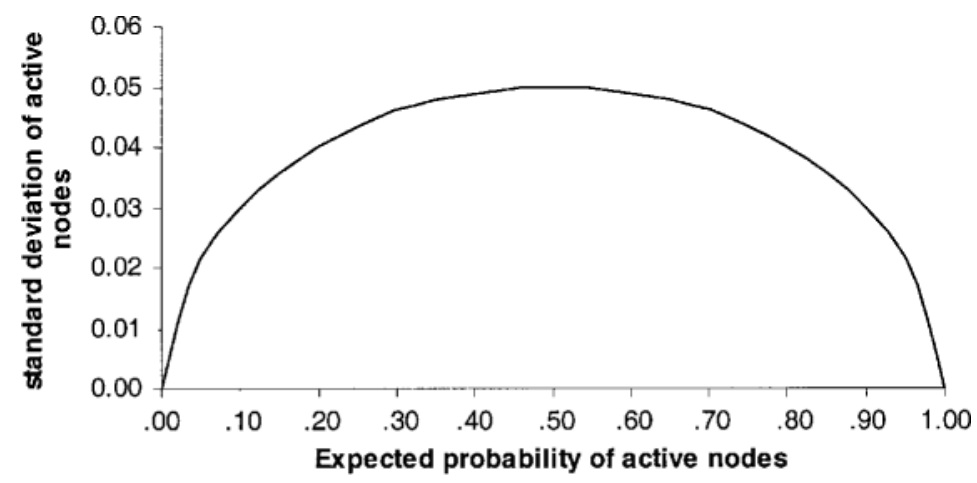

Figure 5. The standard deviation of active nodes as a function of the expected probability that the nodes are active. The standard deviation is calculated with $2 N=100$.

To calculate the recognition strength $(S)$ in Equation 5, the expected percentage of active nodes is subtracted from the percentage of nodes active at recognition $\left(P_{c}\right)$. This is necessary for the normalization to work properly. Owing to the placement of the activation threshold, the expected percentage of active nodes at recognition is half of the expected percentage of nodes active at encoding $(a / 2$; see note 1$)$. This is a constant independent of item class, new or old item, and test difficulty. The result is divided by the standard deviation of the net inputs associated with nodes active at encoding $\left(\sigma_{h}^{\prime}\right)$.

Note that the standard deviation of the net inputs of the to-be-recognized item $\left(\sigma_{h}^{\prime}\right)$ varies on an item-to-item basis around the standard deviation of the net inputs of all items in the class $\left(\sigma_{h}\right)$. This fluctuation may be so large that it is not possible to accurately sort the words into classes on the basis of the standard deviation of the items; however, there is no need for the subject to make such classification in the variance model. The subjects do not need to know the true standard deviation of net inputs in the class. A yes response occurs if the recognition strength is larger than or equal to the subject's recognition criterion $(C$; i.e., if $S \geq C$ ). A no response occurs if the recognition strength is less than the subject's recognition criterion $(S<C)$.

The standard deviation of the net inputs does not affect the probability of a yes response when the recognition criterion is unbiased $(C=0)$. In this special case, the recognition strength can be simplified to $S=P_{c}$ where $C=a / 2$. The standard deviation of the net inputs in Equation 5 affects the probability of a yes response when the recognition criterion is biased $(C \neq 0)$. Thus, the standard deviation of the net inputs in Equation 5 may be interpreted as a scaling factor that influences the confidence measurement (but not the unbiased recognition measurements). A large standard deviation of the net input for an item (correlated with difficulty) influences the decision toward uncertainty, whereas a small standard deviation of the net input for an item (correlated with less difficulty) influences the decision to be more certain.
Figure 4D shows the density distribution of the recognition strength. Note how the standard deviation of the active nodes for the easy class versus the difficult classes (in Figure 4C) changes when it is normalized by the variance of the net input (in Figure 4D). The normalization factor makes the standard deviation of the recognition strength of the difficult class smaller than that of the easy class. Thus, the standard deviation of the recognition strength is proportional to the inverse of the standard deviation of the net input. The difficult class items yield a small standard deviation of the recognition strength, because the standard deviation of the net inputs is high. The easy class items yield a large standard deviation of the recognition strength, because the standard deviation of the net inputs is small. The ordering of the means of the distributions is unaffected by the normalization, and the normalization does not change the fact that the old distributions have a larger standard deviation than do the new distributions.

\section{PREDICTIONS}

This section describes the predictions of the variance theory. We have just seen that the variance theory predicts a material-based mirror effect for high- and low-frequency items because the low-frequency items have a smaller variance of net inputs. This yields lower false alarm rates and higher hit rates for the easy than for the difficult class when the activation threshold is set between the new and the old distributions. Here, it is shown how the model accounts for other effects, such as the strengthbased mirror effect between lists, list-length effects, and the shift in the response criterion. Most important, the variance theory makes predictions regarding the strengthbased mirror effect within lists that is different from the predictions of the attention-likelihood theory. An experiment is conducted to test these predictions. Comparative modeling fitting was also conducted to compare the variance theory with the attention-likelihood theory. The predictions of the theory are based on an analytic solution 
that is presented at the end of the paper, together with an analysis of optimal performance.

\section{The Material-Based Mirror Effect for High- and Low-Frequency Items}

The variance theory was simulated above; here, the analytical results are presented. The variance theory predicts the mirror effect for any choice of parameters when the recognition criterion is unbiased. As will be discussed later, the variance theory can be fully described by two parameters (the number of nodes, $N$, and the percentage of active nodes, $a$ ) plus one parameter for each class or words [the standard deviation of the net input, $\sigma_{h}()$ ]. The following parameters are used here: The number of nodes is $2 N=100$, and the percentage of nodes active at encoding is set to $a=.1$. The standard deviation of the net inputs to the easy class is $\sigma_{h}(\mathrm{AN})=\sigma_{h}(\mathrm{AO})=1.25$, and the standard deviation of the net inputs to the difficult class is $\sigma_{h}(\mathrm{BN})=\sigma_{h}(\mathrm{BO})=1.56$. There are other parameters, which, however, as will be discussed later, do not add any degrees of freedom to the model: the expected net inputs of the new distributions, $\mu_{h}(\mathrm{AN})=$ $\mu_{h}(\mathrm{BN})=0$, and the expected net inputs of the old distributions, $\mu_{h}(\mathrm{AO})=\mu_{h}(\mathrm{BO})=1$. Consequently, the activation threshold is $T=0.5$.

These parameters yield the following probabilities that a node is active at recognition: $P_{c}(\mathrm{AN})=.43 a, P_{c}(\mathrm{BN})=$ $.45 a, P_{c}(\mathrm{BO})=.55 a, P_{c}(\mathrm{AO})=.57 a$. The following expected recognition strengths are predicted: $\mu_{s}(\mathrm{AN})=$ $-0.012, \mu_{s}(\mathrm{BN})=-0.008, \mu_{s}(\mathrm{BO})=0.008, \mu_{s}(\mathrm{AO})=$ 0.012 . Figure $4 \mathrm{D}$ plots the four recognition strength densities (the distributions are assumed to be normal), using the parameters above. The same parameter settings were used in Figures 4A, 4B, 4C, and 5.

\section{Strength-Based Mirror Effects Between Lists}

The variance theory is consistent with the strengthbased mirror effects. Thus, variables that increase the hit rates also decrease the false alarm rates. This empirical finding is called dispersion, which means that the new and the old distributions move apart. The opposite phenomenon is called concentering, which means that the new and the old distributions move closer together. Examples of variables showing strength-based mirror effects are speed versus accuracy instructions, length of study time, encoding task, forgetting, repetition, and aging (Kim \& Glanzer, 1993). These experimental variables can be related to a specific parameter in the variance theory-namely, the expected net input.

The variance theory predicts a strength-based mirror effect because subjects must adjust the activation threshold to optimize the performance. This change in activation threshold affects the false alarm rates. For example, assume that study time is increased from 1 to $2 \mathrm{sec}$, so that the expected net input increases from 1 to 2 and the activation threshold increases from $1 / 2$ to 1 . This diminishes the false alarm rate. However the increase in the activation threshold is smaller than the increase in the old net input, so the hit rate will increase. Thus, increasing the study time increases the hit rate but decreases the false alarm rate, which is dispersion.

The mirror effect is accounted for in some theories by a change in the recognition criterion. Note that in the variance theory, the recognition criterion is constant, whereas the activation threshold is changed. There is an important difference between a change in the recognition criterion and a change in the activation threshold. The change in the activation threshold optimizes the performance as measured by $d^{\prime}$, whereas a change in the recognition criterion does not influence $d^{\prime}$. Given an optimal placement of the activation threshold, the performance in terms of percentage correct is optimal if the recognition criterion is set to an optimal value, which is zero. Thus, there is a clear difference between changing the recognition criterion and changing the activation threshold. The variance theory accounts for the strength-based mirror effect occurring between two conditions by the change in the activation threshold necessary for optimal performance, whereas the recognition criterion does not change.

Concentering occurs, for example, when subjects are instructed to emphasize speed (rather than accuracy), with superficial (rather than deep or semantic) study instructions, with diminished study time, or with an increased retention interval (Kim \& Glanzer, 1993). In the variance theory, all these manipulations are assumed to diminish the old net inputs. Figure 6A shows the predictions of the variance theory when the expected net inputs of the old distributions are $\mu_{h}(\mathrm{AO})=\mu_{h}(\mathrm{BO})=0.5$, rather than 1 as in Figure 4D. Consequently, the activation threshold must be set to 0.25 for optimizing the performance. The distributions in Figure 6A are closer than the distributions in Figure 4D. Thus, decreasing the net inputs-for example, by diminishing study time-moves the distributions closer together, thus showing concentering.

The opposite phenomenon to concentering is dispersion, which means that increasing the performance moves the distributions apart. Dispersion can be studied by changing the variables listed above in the opposite directions-for example, by increasing the study time. Figure $6 \mathrm{~B}$ shows the predictions of the theory when the expected net inputs of the old distributions are $\mu_{h}(\mathrm{AO})=$ $\mu_{h}(\mathrm{BO})=2$. Consequently, the activation threshold must be set to 1 to maintain a near-optimal performance. The distributions in Figure 6B are further apart than the distributions in Figure 4D.

These strength-based manipulations are usually applied between different lists or conditions. For example, Kim and Glanzer (1993) manipulated study time between four study lists, where the items were presented for $1 \mathrm{sec}$ each in two lists and for $2 \mathrm{sec}$ each in two lists. After each list, there was a recognition test. In the variance theory, the activation threshold is the same during each recognition test but may vary between two recognition tests with different levels of difficulty - for example, different study times. As will be discussed later, different empirical results are found when study time is varied within one list. 

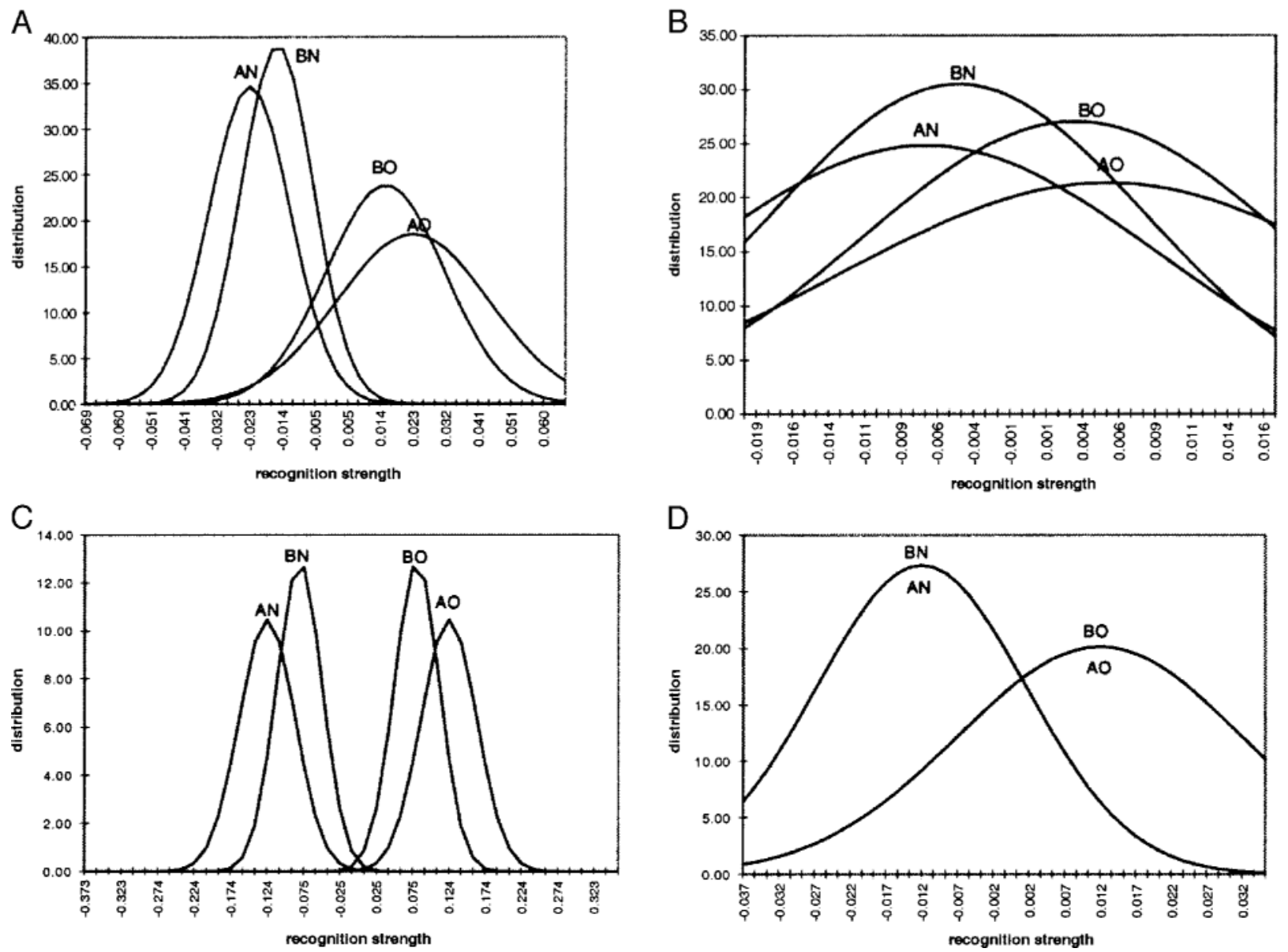

Figure 6. The densities of recognition strength in the variance theory for different parameter settings: (A) concentering, (B) dispersion, (C) activity level set to one, and (D) equal variance. The horizontal axes show the recognition strength, and the vertical axes the density.

In this case, the activation threshold is also constant during the recognition tests, although the study time varies within the condition.

The order of the probabilities in the mirror effect is somewhat robust against changes in the activation threshold over a large range. Setting the activation to a fixed, sufficiently low, and positive value yields the mirror effect for any value of the expected net input. For example, assume that the activation threshold is fixed to 0.4 . Then, the mirror effect is predicted for the three cases of expected old net inputs discussed above $(0.5,1$, and 2$)$ or any value above 0.4 . The predictions for the new distributions do not change with these changes in net inputs $[P(\mathrm{AN})=.25, P(\mathrm{BN})=.30]$; thus, a change in the activation threshold is needed to change the false alarm rates. In contrast, the advantage of the old easy class over

Table 1

General Table of Results From the Experiment

\begin{tabular}{lcccccc}
\hline Condition & $\mathrm{AN}$ & $\mathrm{BN}$ & $\mathrm{BO}$ & $\mathrm{AO}$ & $\sigma_{s}(\mathrm{BN}) / \sigma_{s}(\mathrm{AN})$ & $\sigma_{s}(\mathrm{BO}) / \sigma_{s}(\mathrm{AO})$ \\
\hline Control & 0.13 & 0.17 & 0.69 & 0.82 & 0.60 & 0.86 \\
Frequency & 0.20 & 0.28 & 0.80 & 0.68 & 1.01 & 0.66 \\
Time & 0.10 & 0.15 & 0.78 & 0.76 & 0.89 & 0.81 \\
\hline
\end{tabular}

Note-The rows show the conditions (control, presentation frequency, and presentation time). The columns show the false alarm rates for low (AN) and high (BN) frequencies and the hit rate for high (BO) and low (AO) frequencies, the slope of the $z-\mathrm{ROC}$ curve for the new low-frequency distribution as a function the new high-frequency distribution $\left[\sigma_{s}(\mathrm{BN}) / \sigma_{s}(\mathrm{AN})\right]$, and the corresponding slope for the old distributions $\left[\sigma_{s}(\mathrm{BO}) / \sigma_{s}(\mathrm{AO})\right]$. 
the old difficult class increases with the expected net input [from $P(\mathrm{BO})=.55$ and $P(\mathrm{AO})=.56$ for $\mu_{h}(\mathrm{O})=$ 0.5 to $P(\mathrm{BO})=.89$ and $P(\mathrm{AO})=.92$ for $\left.\mu_{h}(\mathrm{O})=2\right]$.

\section{List-Length Effect}

Given everything else equal, recognition from a short list length has a higher hit rate and lower false alarm rate than recognition from a long list. In the variance theory, list length is predicted to affect the standard deviation of the net input $\left(\sigma_{h}\right)$ for both easy and difficult class items, so that longer lists have a larger variance than do shorter lists. The expected value of the net input is not affected by list length.

Assume that context does not change within a list but is uncorrelated between different lists. The context for a list is thus associated with as many items as there are items in the list. The variance of the net inputs to the item nodes increases when the list length is increased. The reason for this increase in variance is essentially the same as the reason that word frequency affects the variance. In the word frequency case, the same item is associated with several contexts, and this increases the variance in context nodes. In the list-length case, the same context is associated with several items, and this increases the variance in the item nodes. Thus, the variance of the net inputs in the item nodes will be a linear function of list length. Therefore, a long list will have a lower hit rate and a larger false alarm rate than will a short list.

\section{ROC Curves}

The percentage of nodes active at recognition is less for new items than for old items. Owing to the placement of the activation threshold, this proportion is always less than $1 / 2$. The standard deviation of the percentage of active nodes increases as a function of the percentage of active nodes. If the percentage of active nodes is zero, the standard deviation obviously is zero. However, this standard deviation increases as the percentage of active nodes increases. This yields a smaller standard deviation for the new distribution (which is associated with a lower percentage of active nodes), as compared with the old distribution $\left[\sigma_{s}(\mathrm{AO})>\sigma_{s}(\mathrm{AN})\right.$ and $\left.\sigma_{s}(\mathrm{BO})>\sigma_{s}(\mathrm{BN})\right]$.

For the sake of understanding the model, the proportion of nodes active at encoding can be set unrealistically high-namely, to $a=1$. This setting yields around $50 \%$ of these nodes being active at recognition. This parameter setting makes the standard deviations of the new and the old distributions equal $\left[\sigma_{s}(\mathrm{AO})=\sigma_{s}(\mathrm{AN})\right.$ and $\sigma_{s}(\mathrm{BO})=$ $\left.\sigma_{s}(\mathrm{BN})\right]$. Figure $6 \mathrm{C}$ shows the prediction for $a=1$ (all the other parameters are identical to those in Figure 4D).

The standard deviation of recognition strength is larger for the difficult class than for the easy class $\left[\sigma_{s}(\mathrm{AN})>\right.$ $\sigma_{s}(\mathrm{BN})$ and $\left.\sigma_{s}(\mathrm{AO})>\sigma_{s}(\mathrm{BO})\right]$, because the recognition strengths are calculated from the inverse of the standard deviation of the net inputs. Thus, when the standard deviations of the net inputs are set equal, the standard deviation of the recognition strengths and the recognition strengths becomes equal. Figure 6D plots the predictions of the theory when all standard deviations of the net in- puts are 1.25. The other parameters are the same as those in Figure 4D.

In Figure 4D, the four standard deviations of the recognition strengths are $\sigma_{s}(\mathrm{AN})=0.015, \sigma_{s}(\mathrm{BN})=0.012$, $\sigma_{s}(\mathrm{BO})=0.015$, and $\sigma_{s}(\mathrm{AO})=0.020$. The ratio of these standard deviations must follow Equation 2. This is also the case with $\sigma_{s}(\mathrm{AO}) / \sigma_{s}(\mathrm{BN})=0.61<0.74=\sigma_{s}(\mathrm{AO}) /$ $\sigma_{s}(\mathrm{AN})<\sigma_{s}(\mathrm{BO}) / \sigma_{s}(\mathrm{BN})=0.78<0.94=\sigma_{s}(\mathrm{BO}) / \sigma_{s}(\mathrm{AN})$.

\section{Changing the Recognition Criterion}

The probability of a yes response $(P)$ for the four classes depends on the recognition criterion $(C)$. Setting $C$ to an unbiased value of 0 yields $P(\mathrm{AN})=.20, P(\mathrm{BN})=$ $.25, P(\mathrm{BO})=.70, P(\mathrm{AO})=.74$. These predicted data are prototypical of experimental data for the mirror effect.

A conservative value of the recognition criterion $(C)$ will not yield the mirror effect. For example, $C=0.016$ yields $P(\mathrm{AN})=.03, P(\mathrm{BN})=.02, P(\mathrm{BO})=.30, P(\mathrm{AO})=$ .43 . Thus, the variance theory predicts that a conservative recognition criterion yields a higher false alarm rate for easy class words than for difficult class words. This prediction is supported by empirical data. Greene (1996) asked subjects to respond yes only if they were sure of their response. Consistent with the prediction, no mirror effect was found.

It follows from the ordering of the distributions in Figure 4D that the theory also predicts the experimental findings in forced recognition $[P(\mathrm{BO}, \mathrm{BN})<P(\mathrm{AO}, \mathrm{BN})$, $P(\mathrm{BO}, \mathrm{AN})<P(\mathrm{AO}, \mathrm{AN}), P(\mathrm{BN}, \mathrm{AN})>.50$, and $P(\mathrm{AO}$, $\mathrm{BO})>.50]$. For the parameters above, the predictions of the theory are $P(\mathrm{BO}, \mathrm{BN})=.79<.81=P(\mathrm{AO}, \mathrm{BN})$, $P(\mathrm{BO}, \mathrm{AN})=.83<.84=P(\mathrm{AO}, \mathrm{AN}), P(\mathrm{BN}, \mathrm{AN})=.59>$ $.50, P(\mathrm{AO}, \mathrm{BO})=.57>.50$.

\section{Within-List Strength Manipulation}

So far, the predictions made by the variance theory are qualitatively (but not quantitatively) equal to those of the attention-likelihood theory. However, there is an exception that differentiates the variance theory from the attention-likelihood theory. The mirror effect is normally studied under experimental conditions in which the difficult and the easy classes are given the same amount of attention-for example, under conditions in which the number of presentations, the study time, and the study instructions are equal for the two classes of words. However, if the number of presentation is larger for the difficult class than for the easy class, different results emerge. Stretch and Wixted (1998b) conducted five experiments in which the basic manipulation was to present high-frequency words five times, whereas the lowfrequency words were presented once. The results did not show a mirror effect, because the hit rates for the high-frequency words were higher than those for the low-frequency words. However, increasing the number of presentations for the high-frequency words did not affect the false alarm rate, so both the false alarm rate and the hit rate were larger for high-frequency words.

The variance theory accounts for this new finding in the following way. The theory assumes that a longer pre- 


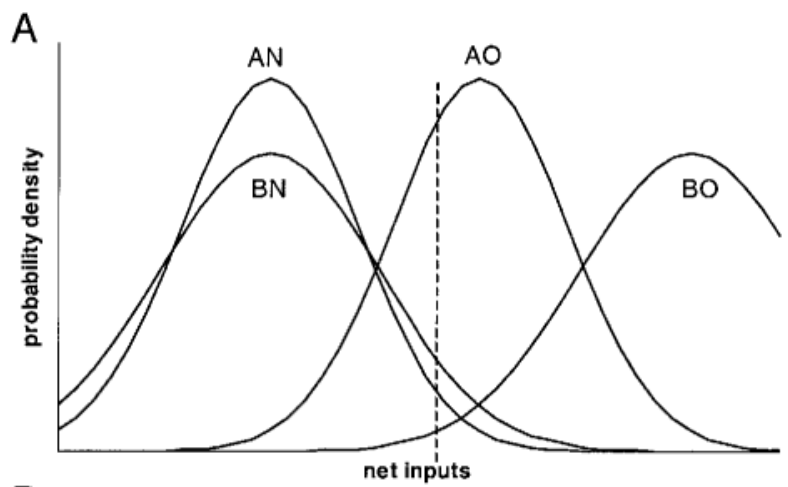

B

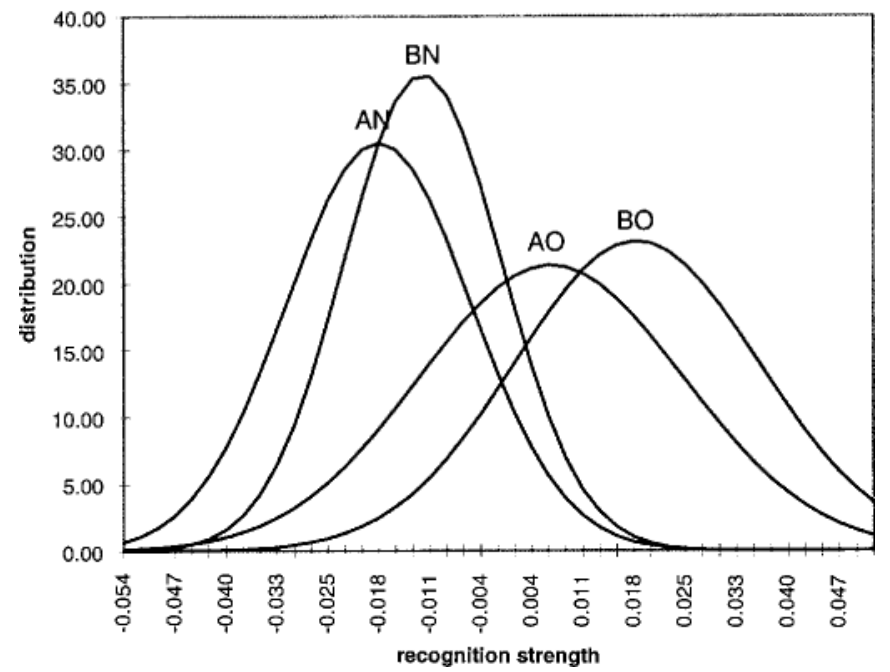

Figure 7. (A) The probability density of the net inputs in the variance theory when attention is focused on the high-frequency class. The horizontal axis shows the net inputs, and the vertical axis the probability density of the net inputs. The expected value of the high-frequency value (BO) is shifted to the right because attention is focused on this class. The dotted vertical line is the activation threshold. (B) The predictions of the variance theory when subjects focus their attention on high-frequency words. The horizontal axis shows the recognition strength, and the vertical axis the probability density.

sentation time, or a larger presentation frequency, increases the net inputs of the old items $\left[\mu_{h}(\mathrm{O})\right]$. This is illustrated in Figure 7A (compare with Figure 4A, where the same amount of attention is paid to the two classes). If the net inputs for old high-frequency items are increased sufficiently, the percentage of active nodes will be larger than that for old low-frequency items. For this to occur, the effect of the increase in net input (which gives the advantage for old high-frequency items when attention is focused on these items) must be larger than the effect from the larger standard deviation of the net inputs for old high-frequency items (which gives the advantage for old low-frequency items when the same attention is paid to the two classes). This increase in the percentage of active nodes yields a higher hit rate for high-frequency items than for low-frequency items.
However, it will not significantly change the false alarm rates, which are larger for high-frequency items than for low-frequency items. Therefore, the variance theory predicts no mirror effect when high-frequency items are presented sufficiently more often or with a sufficiently longer presentation time, as compared with low-frequency items.

It is apparent from the density of net inputs (Figure 7A) that the density of recognition strengths (Figure 7B) will not show a mirror effect (i.e., because the percentage of active nodes are larger for high- than for lowfrequency old items). The parameters used in these figures are identical to the parameters used for the standard mirror effect in Figures 4A and 4D, with the exception that the expected net input of the old high-frequency items $\left[\mu_{h}(\mathrm{BO})\right]$ is 2 rather than 1 . Consequently, to optimize performance, the activation threshold becomes 
0.75 rather than 0.50 . The figure does not show a mirror effect, because the expected hit rate and the expected false alarm rate are larger for the high-frequency items than for the low-frequency items. Setting $C$ to an unbiased value of 0 yields $P(\mathrm{AN})=.08, P(\mathrm{BN})=.14, P(\mathrm{BO})=$ $.86, P(\mathrm{AO})=0.63$ [which may be compared with Figure $6 \mathrm{~B}: P(\mathrm{AN})=.20, P(\mathrm{BN})=.25, P(\mathrm{BO})=.70, P(\mathrm{AO})=.74]$.

Furthermore, in the variance theory, the ratio of the recognition strength standard deviations for high- and low-frequency items depends mainly on the standard deviations of the net inputs. The standard deviations of the net inputs are not dependent on the attention paid to the stimuli. Therefore, the variance theory predicts no change in the standard deviations when the amount of attention is manipulated. The standard deviation of the old lowfrequency distribution is predicted to be larger than the standard deviation of the old high-frequency distribution. Similarly, the standard deviation of the new lowfrequency distribution is predicted to be larger than that of the new high-frequency distribution. The standard deviations in Figure 7B are $\sigma_{s}(\mathrm{AN})=0.013, \sigma_{s}(\mathrm{BN})=0.011$, $\sigma_{s}(\mathrm{BO})=0.017$, and $\sigma_{s}(\mathrm{AO})=0.019$. These results are similar to the results when the same amount of attention is paid to the two classes in Figure 4D: $\sigma_{s}(\mathrm{AN})=0.015$, $\sigma_{s}(\mathrm{BN})=0.012, \sigma_{s}(\mathrm{BO})=0.015$, and $\sigma_{s}(\mathrm{AO})=0.020$.

The standard version of the attention-likelihood theory has problems accounting for the lack of mirror effect when more study time is given to the difficult class than to the easy class. This theory suggests that the class of items to which more attention is being paid is more easily recognized. For example, low-frequency items are better recognized than high-frequency items because subjects pay more attention to them. The amount of attention is assumed to influence the number of sampled feature $[n(i)]$, so more features are sampled for low- than for high-frequency items (Kim \& Glanzer, 1993). This is the only parameter that differs between high- and lowfrequency items. From this explanation, it follows that, if the experimental conditions are manipulated so that subjects pay more attention to the high-frequency items, the standard version of the attention-likelihood theory will predict a mirror effect where the high-frequency items are the easier class (A) and the low-frequency items are the more difficult class (B). The difference from the normal mirror effect is a larger hit rate and a smaller false alarm rate for high- than for low-frequency items. Furthermore, the attention-likelihood theory makes predictions of the order of the slope of ROC curves. The standard deviation of the hit rate for the high-frequency distribution would be larger than the hit rate for the low-frequency distribution. Similarly, it is predicted that the standard deviation of the high-frequency false alarm distribution is larger than that of the low-frequency distribution.

\section{EXPERIMENT}

An experiment was conducted to test the predictions regarding the within-list strength manipulation. The number of presentations and the study time of the highfrequency words were manipulated in an experiment. The original rationale for the experiment was to compare the results with the predictions of the variance theory and the attention-likelihood theory, because the experiment was conducted before the publication of Stretch and Wixted's (1998b) study, which manipulated attention by the number of presentations. In this experiment, a new manipulation is investigated-namely, how the amount of study time per item for each class influences the mirror effect. Furthermore, the manipulation of the number of presentations is replicated. Thus, there were two experimental conditions: one in which the amount of study time was manipulated and one in which the presentation time was manipulated. There was also one control condition in which high- and low-frequency words were given the same amount of attention.

\section{Method}

Subjects. Twenty-one students taking the introductory psychology course at the University of Toronto volunteered to participate in a memory experiment for course credit. There were 14 female and 7 male subjects, with a mean age of 20 , ranging from 18 to 29 years old.

Material. Sixty low-frequency words and 60 high-frequency words were selected from Kučera and Francis (1967). The lowfrequency words have an occurrence of 4-5 times per million and the high frequency words an occurrence of 50-55 times per million. Thirty low- and 30 high-frequency words were randomly chosen for List 1, and the remaining for List 2.

Procedure. The subjects were instructed to study a list of words so they would be able to recognize the words after study. Fifteen low-frequency words and 15 high-frequency words were randomly chosen as study words for each subject.

Design. There were three conditions. In all the conditions, the low-frequency words were presented once with a presentation time of $1 \mathrm{sec}$. In the control condition, the high-frequency words were also presented once with a presentation time of $1 \mathrm{sec}$. In the presentation frequency condition, the high-frequency words were presented twice for $1 \mathrm{sec}$ each time. In the presentation time condition, the high-frequency words were presented once for $3 \mathrm{sec}$. The presentation order was randomized. All the words were presented in uppercase on a blank computer screen. Immediately following the study list, there was a recognition test. The subjects were presented with either one of the studied words or one of the lures. There were 15 low-frequency lures and 15 high-frequency lures in each condition. The subjects were asked to judge whether the word was presented in the list or not. The subjects were also required to rate their confidence in their responses on a scale ranging from 1 (guessing) to 5 (very certain). The order of recognition was randomized for each subject.

Each subject participated in two conditions. List 1 was always given as the first list, and List 2 as the second list. Twelve subjects were randomly chosen for the presentation frequency condition followed by the presentation time condition. Nine subjects were given the control condition followed by another control condition. The whole experimental set-up, including instructions, presentation of words, and the recognition test, was automated on a computer. Each subject was tested individually.

\section{Results}

The results from the experiment are presented in the first three rows of Table 1 . The probability for hit rates 
was larger for the high-frequency words than for the lowfrequency words in the presentation frequency and the presentation time conditions. In the control condition, the probability for hit rates for the low-frequency condition was larger. One-tailed paired $t$ tests over the performance for each subject were carried out to test the differences between the high and the low frequencies. The effects were significant in the presentation frequency condition $\left[t(11)=2.2, M S_{\mathrm{e}}=0.04, p=.02<.05\right]$ and in the control condition $\left[t(16)=-3.3, M S_{\mathrm{e}}=0.04, p=.00\right.$ $<.05]$, but not in the presentation time condition $[t(11)=$ $\left.0.41, M S_{\mathrm{e}}=0.03, p=.34<.05\right]$.

The false alarm rate was larger for the high-frequency words in all the conditions. However, it was only significantly larger in the presentation frequency condition $\left[t(11)=-1.8, M S_{\mathrm{e}}=0.03, p=.048<.05\right]$, but not in the presentation time condition $\left[t(11)=-1.5, M S_{\mathrm{e}}=0.01, p=\right.$ $.07>.05]$ and the control condition $\left[t(16)=-1.4, M S_{\mathrm{e}}=\right.$ $0.02, p=.09>.05]$.

The results in the presentation frequency condition support the variance theory. The hit and the false alarm rates were significantly larger for the high-frequency words than for the low-frequency words. Thus, there was no mirror effect. However, the prediction of the standard version of the attention-likelihood theory was not supported.

The results in the presentation time condition were in the same direction as those in the presentation frequency condition, although the difference between the high and the low frequencies was not significant. This condition is consistent with the variance theory, although the standard version of the attention-likelihood theory could not be dismissed in this condition, since the results were nonsignificant.

Finally, the control condition yielded results consistent with previous studies showing a mirror effect. The hit rate for the high-frequency words was significantly lower than the hit rate for the low-frequency words. The false alarm rate for the high-frequency words was larger than that for the low-frequency words (although not significantly). Thus, the control condition is, as was expected, consistent with both the variance theory and the standard version of the attention-likelihood theory.

The slopes of the ROC curves were calculated as follows. The hit and false alarm rates for confidence ratings 1-5 were $z$-transformed (e.g., for confidence rating 4 , a hit response was scored if the confidence rating was 4 or above). Linear regressions of one $z$-transformed measurement as a function of another $z$-transformed measurement were conducted. The slope of the linear regression curves between the $z$-transformed hit rate of the lowfrequency words and the $z$-transformed hit rate of the highfrequency words $\left[\sigma_{s}(\mathrm{BO}) / \sigma_{s}(\mathrm{AO})\right]$, and similarly for the slope of the false alarms $\left[\sigma_{s}(\mathrm{BN}) / \sigma_{s}(\mathrm{AN})\right]$, are shown in the last two rows of Table 1.

The results show that the standard deviations of the old high-frequency distributions were smaller than the standard deviations of the old low-frequency distributions in all the conditions. The standard deviations of the false alarm high-frequency distributions were smaller than the standard deviations of the false alarm lowfrequency distributions in the presentation frequency condition and the control condition but were approximately equal in the presentation time condition.

To summarize, the results in the presentation frequency condition are consistent with the variance theory and inconsistent with the standard version of the attentionlikelihood theory. The control condition is consistent with both the variance theory and the standard version of the attention-likelihood theory. These data are also consistent with results presented by Stretch and Wixted (1998b). However, Stretch and Wixted (1998b) suggested one possible way to modify the standard version of the attention-likelihood theory to bring it in line with the data presented here. They noted that Glanzer et al. (1993) had shown that the attention-likelihood theory predicts the mirror effect although $p(i$,old $)$ is set to the average value of the two classes. This modified version can predict the pattern of data presented here, given that the attention paid to the high-frequency class was high during encoding $[n(\mathrm{~B})=120]$ and low during recognition $[n(\mathrm{~B})=$ 40]. This formulation of the attention-likelihood theory seems somewhat unclear. It is not well motivated why $p(i$, old ) should be equal during recognition, whereas $n(i)$ is different $[p(i$,old $)$ is calculated from $n(i)]$, or why the amount of attention for high-frequency items is lower than that for low-frequency words at encoding, but higher at recognition.

\section{COMPARATIVE DATA FITTING}

Glanzer et al. (1993) fitted the attention-likelihood theory to experimental data in nine conditions. The easy class (A) consisted of either low-frequency words or concrete words, and the difficult class (B) consisted of high-frequency words or abstract words. Here, the variance theory is also fitted to the same set of data as that used in Glanzer et al. (1993). This allows a direction evaluation of the variance theory by comparing its fits with those of the attention-likelihood theory.

Glanzer et al. (1993) fitted the attention-likelihood theory to the four probabilities of yes responses and the four ratios of slopes of the ROCs. The fitting was conducted by minimizing the mean squared error divided by the variance-that is,

$$
\sum_{i=1}^{8} \frac{\left(\text { Observed }_{i}-\text { Predicted }_{i}\right)^{2}}{\sigma_{i}^{2}} .
$$

Three parameters were fitted-namely, the attention paid to each of the classes, $n(\mathrm{~A})$ and $n(\mathrm{~B})$, and the probability that a feature was activated, $p$ (new). The other parameters were held constant at a value found to give a good fit. These parameters were $(N=1,000)$ and the recognition criterion $[\ln (L)=0]$.

The variance theory was fitted to the same set of data, using the same technique and the same number of free parameters. The fitted parameters were the percentage 
A

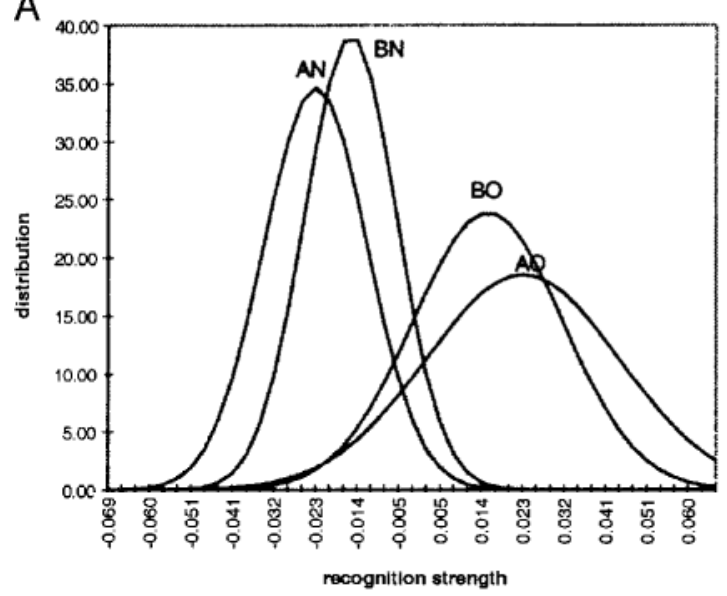

C

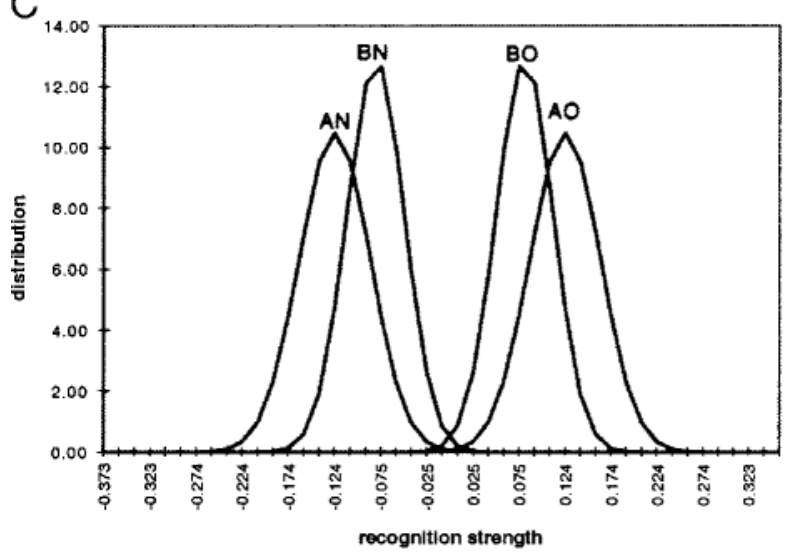

B

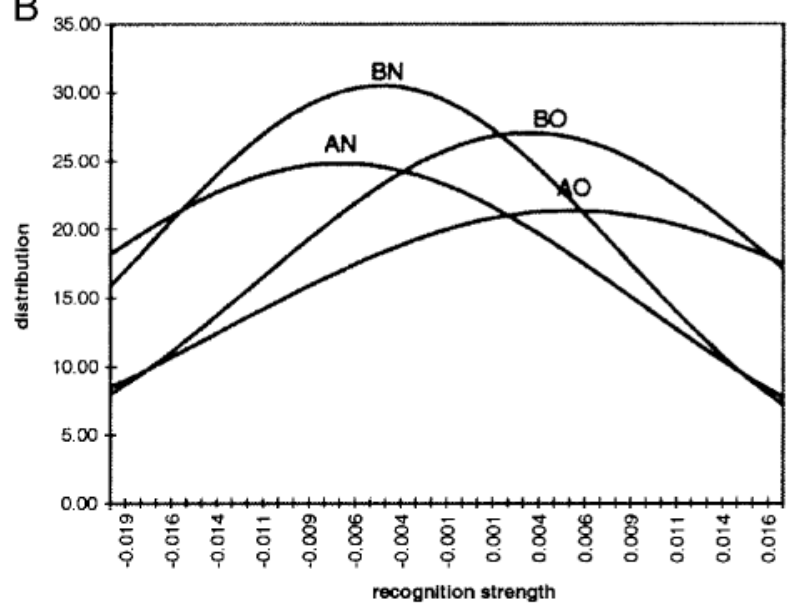

D

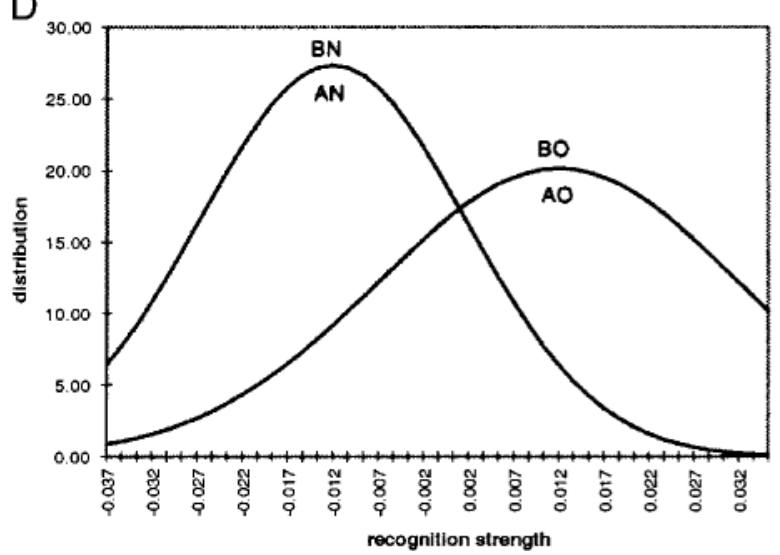

Figure 6. The densities of recognition strength in the variance theory for different parameter settings: (A) concentering, (B) dispersion, (C) activity level set to one, and (D) equal variance. The horizontal axes show the recognition strength, and the vertical axes the density.

In this case, the activation threshold is also constant during the recognition tests, although the study time varies within the condition.

The order of the probabilities in the mirror effect is somewhat robust against changes in the activation threshold over a large range. Setting the activation to a fixed, sufficiently low, and positive value yields the mirror effect for any value of the expected net input. For example, assume that the activation threshold is fixed to 0.4 . Then, the mirror effect is predicted for the three cases of expected old net inputs discussed above $(0.5,1$, and 2$)$ or any value above 0.4 . The predictions for the new distributions do not change with these changes in net inputs $[P(\mathrm{AN})=.25, P(\mathrm{BN})=.30]$; thus, a change in the activation threshold is needed to change the false alarm rates. In contrast, the advantage of the old easy class over

Table 1

General Table of Results From the Experiment

\begin{tabular}{lcccccc}
\hline Condition & $\mathrm{AN}$ & $\mathrm{BN}$ & $\mathrm{BO}$ & $\mathrm{AO}$ & $\sigma_{s}(\mathrm{BN}) / \sigma_{s}(\mathrm{AN})$ & $\sigma_{s}(\mathrm{BO}) / \sigma_{s}(\mathrm{AO})$ \\
\hline Control & 0.13 & 0.17 & 0.69 & 0.82 & 0.60 & 0.86 \\
Frequency & 0.20 & 0.28 & 0.80 & 0.68 & 1.01 & 0.66 \\
Time & 0.10 & 0.15 & 0.78 & 0.76 & 0.89 & 0.81 \\
\hline
\end{tabular}

Note-The rows show the conditions (control, presentation frequency, and presentation time). The columns show the false alarm rates for low (AN) and high (BN) frequencies and the hit rate for high (BO) and low (AO) frequencies, the slope of the $z$-ROC curve for the new low-frequency distribution as a function the new high-frequency distribution $\left[\sigma_{s}(\mathrm{BN}) / \sigma_{s}(\mathrm{AN})\right]$, and the corresponding slope for the old distributions $\left[\sigma_{s}(\mathrm{BO}) / \sigma_{s}(\mathrm{AO})\right]$. 
probabilities. Figure $8 \mathrm{~B}$ shows the corresponding results for the slope. The accounted variance is 0.96 for the probabilities and 0.85 for the slopes. Thus, the variance theory fits the slopes, using a single parameter, equally well as the attention-likelihood theory does with three fitting parameters. The fit for the variance theory for the probabilities, using one parameter, is slightly less than the fit of the attention-likelihoodtheory, using three fitting parameters. It may be concluded that the fit for the variance theory is reasonably good for the probabilities and the slopes. The slopes have a better fit in the variance theory than in the attention-likelihood theory when three variables are used.

\section{ANALYTIC SOLUTIONS}

In this section, analytic solutions of the variance theory, an approximation of the standard deviation of the recognition strength, and analyses of optimal performance are presented. The variance theory has a simple analytic solution and can be fully described by four parameters. Two of these parameters-namely, the standard deviation of the net inputs from the easy class $\left[\sigma_{h}(\mathrm{~A})\right]$ and the standard deviation of the net inputs from the difficult class $\left[\sigma_{h}(\mathrm{~B})\right]$ - can also be expressed as the frequency of the item (see the Appendix). The other two parameters are the number of nodes $(N)$ and the expected probability that the nodes are active at encoding $(a)$.

There are other variables in the theory; however, they do not increase the degrees of freedom. There are four expected net inputs $\left(\mu_{h}\right)$; however, two degrees of freedom disappear because the new net inputs are constrained to be equal, as well as the old net inputs $\left[\mu_{h}(\mathrm{AN})=\right.$ $\mu_{h}(\mathrm{BN})$ and $\left.\mu_{h}(\mathrm{AO})=\mu_{h}(\mathrm{BO})\right]$. Furthermore, the predictions are independent of moving the old and new distributions in parallel, thus removing another degree of freedom. Finally, changing the difference between the expected old and new net inputs is mathematically equivalent to changing the standard deviations $\left[\sigma_{h}(\mathrm{~A})\right.$ and $\left.\sigma_{h}(\mathrm{~B})\right]$. Thus, the degrees of freedom in the net inputs can be captured by the degrees of freedom in the standard deviations. The activation threshold $(T)$ and the probability that nodes are active $\left(P_{\mathrm{c}}\right)$ are simply functions of other variables and, therefore, do not increase the degrees of freedom. Thus, there are four degrees of freedom for the distributions - namely, $\sigma_{h}(\mathrm{~A}), \sigma_{h}(\mathrm{~B}), N$, and $a$. An additional degree of freedom is introduced when placing the recognition criterion $(C)$.

The probability $\left(P_{c}\right)$ that the net inputs exceed the activation threshold $(T)$ for nodes active during encoding can be explicitly solved from the expected net inputs $\left(\mu_{h}\right)$ and the standard deviation of the net inputs $\left(\sigma_{h}\right)$. This probability is dependent on the distribution of the net inputs, which can be approximated with a normal distribution. $P_{c}$ is solved by integrating the net inputs from $\mu_{h}-T$ to infinity ( $\infty$ ) over the probability density function for a normal distribution. Thus, the probability $\left(P_{\mathrm{c}}\right)$ that a node is active at recognition is

$$
P_{c}=a \frac{1}{\sqrt{2 \pi}} \int_{T}^{\infty} e^{\frac{\left(h-\mu_{h}\right)^{2}}{2 \sigma_{h}^{2}}} d h .
$$

Subtracting the expected percentage of active nodes at recognition $(a / 2$; see note 1$)$ from the percentage of active nodes and dividing by the standard deviation of the net inputs $\left(\sigma_{h}\right)$ calculates the expected recognition strength $\left(\mu_{s}\right)$ :

$$
\mu_{s}=\frac{P_{c}-\frac{a}{2}}{\sigma_{h}} .
$$

Note that the analytic solution uses the standard deviation of the class $\left(\sigma_{h}\right)$ as an approximation of the standard deviation of the item $\left(\sigma_{h}^{\prime}\right)$, because it simplifies the analytic solution; however, the variance theory or the simulation uses the standard deviation of the item. This approximation is good when there are a large number of features; however, for a small number of features, the variance of feature strength for a single item may fluctuate on an item-to-item basis around the variance of the net inputs for all the items.

The standard deviation of the recognition strength $\left(\sigma_{s}\right)$ is calculated from $\sigma_{h}, P_{c}$, and $N$. There is $2 N$ number of nodes in the context and the item layers. The distribution of $P_{c}$ is binomial but can, for a certain criterion [i.e., $2 N$ $\left.P_{c}\left(1-P_{c}\right)>10\right]$, be approximated with a normal distribution with a standard deviation of $\left[P_{c}\left(1-P_{c}\right) / 2 N\right]^{1 / 2}$. The final result is scaled by the normalization factor $1 / \sigma_{h}$ :

$$
\sigma_{s}=\frac{1}{\sigma_{h}}\left[\frac{P_{c}\left(1-P_{c}\right)}{2 N}\right]^{-\frac{1}{2}}
$$

A yes response occurs if the recognition strength is above the recognition criterion $(C)$. The probability of a yes response $[P(\mathrm{Y})]$ is calculated from the expected recognition strength, the variance of the recognition strength, and the recognition criterion by integrating the density of the recognition strength over a normal distribution:

$$
P(\mathrm{Y})=\frac{1}{\sqrt{2 \pi}} \int_{C}^{\infty} e^{\frac{\left(s-\mu_{s}\right)^{2}}{2 \sigma_{s}^{2}}} d s .
$$

The probability of choosing A over B in a two-choice forced recognition test $[P(\mathrm{~A}, \mathrm{~B})]$ is calculated from the expected recognition strength of $\mathrm{A}\left[\mu_{s}(\mathrm{~A})\right]$ and $\mathrm{B}\left[\mu_{s}(\mathrm{~B})\right]$ and the standard deviations of the recognition strength of $\mathrm{A}\left[\sigma_{s}(\mathrm{~A})\right]$ and $\mathrm{B}\left[\sigma_{s}(\mathrm{~B})\right]$ :

$$
P(\mathrm{~A}, \mathrm{~B})=\frac{1}{\sqrt{2 \pi}} \int_{C}^{\infty} e^{\frac{\left(s-\left[\mu_{s}(\mathrm{~A})-\mu_{s}(\mathrm{~B})\right]\right)^{2}}{2\left[\left(\sigma_{s}^{2}(\mathrm{~A})^{2}+\sigma_{s}(\mathrm{~B})^{2}\right)\right.}} d s .
$$

An Excel sheet for calculating the predictions of the variance theory is available on line (www.psych.utoronto. ca/ sverker/variance.html). 


\section{Approximations of the Standard Deviation of Recognition Strength}

The standard deviation of the recognition strength is in the model calculated with Equation 7. However, to facilitate the understanding of this equation, it is useful to make some approximations. First, note that the probability that a node is active $\left(P_{c}\right)$ is assumed to be low. By approximating $1-P_{c}$ to one, the variance of the recognition strength can be simplified to

$$
\sigma_{s}^{2}=\frac{P_{c}}{\sigma_{h}^{2} 2 N} .
$$

For a particular class of items, the variances of the net inputs of old and new items are equal, and the variance of the recognition strength is proportional to the number of active nodes $\left(\sigma_{s}^{2} \propto P_{c}\right)$. This approximation suggests a very simple interpretation of the slope of the $z$-ROC. The ratio of variances between new and old items is simply the ratio between the number of nodes active in the new items representations and the number of nodes active in the old items representations:

$$
\frac{\sigma_{s}^{2}(\mathrm{~N})}{\sigma_{s}^{2}(\mathrm{O})} \approx \frac{P_{c}(\mathrm{~N})}{P_{c}(\mathrm{O})} .
$$

Or alternatively, the slope of the $z$-ROC curve is equal to the square root of the ratio of the number of nodes active in the new items representations and the number of nodes active in the old items representations. For example, if the slope of the $z$-ROC curve is 0.8 , the number of active nodes in the new items representations divided by the number of nodes active in the old items representations is $0.64\left(=0.80^{2}\right)$.

Another approximation useful for understanding the model is that, for two classes of items, the number of active nodes in the new distribution is approximately equal, and the number of active nodes in the old distributions is approximately equal $\left[P_{c}(\mathrm{AN}) \approx P_{c}(\mathrm{BN})\right.$ and $P_{c}(\mathrm{BO}) \approx$ $\left.P_{c}(\mathrm{AO})\right]$. Given these approximations and the approximation above $\left(1-P_{c} \approx 1\right)$, the recognition strength standard deviation is inversely related to the standard deviation of the net inputs in the following way. The ratio between the recognition strength standard deviations of the difficult and the easy distributions is equal to the ratio between the standard deviations of the net inputs of the easy and the difficult distributions. Furthermore, the ratio between the recognition strength standard deviations of the difficult and easy new distributions is equal to the ratio between the recognition strength standard deviations of the difficult and the easy old distributions. The exact solution predicts a slightly smaller ratio in the old than in the new distributions:

$$
\frac{\sigma_{s}(\mathrm{BO})}{\sigma_{s}(\mathrm{AO})} \leq \frac{\sigma_{s}(\mathrm{~B})}{\sigma_{s}(\mathrm{~A})} \approx \frac{\sigma_{h}(\mathrm{~A})}{\sigma_{h}(\mathrm{~B})} \leq \frac{\sigma_{s}(\mathrm{BN})}{\sigma_{s}(\mathrm{AN})} .
$$

This suggests that the ratio between the recognition strength standard deviations of the difficult class and the easy class can be interpreted as the ratio between the standard deviations of the net inputs of the easy class and the difficult class.

\section{Optimizing Performance Derives the \\ Assumptions of the Variance Theory}

Arguably, good memory performance is an important factor for selection in the evolutionary process of humans and animals. It is reasonable to assume that the brain has evolved so that the performance at retrieval is optimal or near-optimal. Here, it is investigated how several assumptions of the variance theory influence performance. It is shown that several assumptions of the model fall out as a consequence of optimizing performance in the form of discriminability between new and old items. Thus, if the model is implemented in a different way, performance is degraded, and the model does not account for the experimental data. Examples of assumptions that yield a good performance in the model are a low percentage of nodes active, setting the activation threshold between old and new net inputs, measuring performance by nodes that are active in the encoding pattern, and normalizing the recognition strength. It is shown that an optimal performance in the network requires the implementation suggested by the variance theory. If the implementation of the variance theory is changed significantly, the performance is degraded, and the network would not produce the empirically found memory phenomena.

To conduct this analysis, it is necessary to define a measurement of performance. A natural choice is to use $d^{\prime}$. By using the analytical equations above, we find the following expression:

$$
d^{\prime}=\frac{\mu_{s}(\mathrm{O})-\mu_{s}(\mathrm{~N})}{\sigma_{s}(\mathrm{NO})}=\frac{P_{c}(\mathrm{O})-P_{c}(\mathrm{~N})}{\left[P _ { c } ( \mathrm { NO } ) \left(1-\left.P_{c}(\mathrm{NO}) \frac{1}{2 N}\right|^{-\frac{1}{2}}\right.\right.} .
$$

Because $\sigma_{s}(\mathrm{~N})$ sometimes is near zero, it was found useful to use the standard deviation of both the old and the new items recognition strength $\sigma_{s}(\mathrm{NO})$ in the denominator of this equation. Thus, $P_{c}(\mathrm{NO})$ is equal to $\left[P_{c}(\mathrm{~N})+P_{c}(\mathrm{O})\right] / 2 . P_{c}()$ was calculated with Equation 6. The expected value of the net inputs and the standard deviation of the net inputs for new and old items were calculated with the equations derived in the Appendix (Equations A1, A2, and A3). Low-frequency items with a preexperimental frequency of zero were used $(f=0)$, and the list length was set to one $(L=1)$.

The performance can be expressed by the parameters $a, N$, and $p$. Increasing the number of nodes $(N)$ monotonically increases $d^{\prime}$, and increasing the number of stored patterns $(p)$ monotonically decreases $d^{\prime}$. The impact of these two parameters on $d^{\prime}$ was of less importance here, and they were set to $N=30$ and $p=100$.

Optimal percentage of nodes active at encoding. The solid line in Figure 9A shows the theoretical $d^{\prime}$ as a function of the percentage of nodes active at encoding 
(a). The results show that $d^{\prime}$ is optimal for $a=.052$. The $d^{\prime}$ is lower for larger and smaller $a$. The lower $d^{\prime}$ for large $a$ occurs because the interference from other items increase. For an $a$ larger than the optimal value, the weight changes are distributed over a larger number of nodes, and the recognition tests therefore include more noise.

The lower $d^{\prime}$ for an $a$ less than the optimal value occurs because there is variability in the number of active nodes at encoding. Thus, for very small values of $a$, the fluctuation between the number of nodes active in the encoded representation becomes increasingly important. Thus, for a small $a$, errors are more likely to occur, because old items have few active nodes at encoding, making it less likely that many nodes will be active at retrieval (independently of how well they are encoded). This analysis suggests that a medium low percentage of active nodes at encoding yields optimal performance. This is consistent with variance theory, which requires a low activity for fitting some of the empirical data (see below).

There is another factor that contributes to the fact that optimal performance occurs when the percentage of active nodes is medium low, which is that the number of possible representations increases with $a$. If there is only one node active in all the representations, there are $N$ possible combinations of representations; if there are two nodes active in all the representations, there are approximately $N^{2}$ possible combinations of representations; and so forth. This factor is not included in the analyses.

Optimal placement of the activation threshold. An important property of $d^{\prime}$ is that it is insensitive to where the criterion is placed. Thus, any criterion yields the same performance. The activation threshold $(T)$ may be seen as the criterion for a single node, and therefore, one might intuitively think that the placement of the threshold is unimportant for $d^{\prime}$. However, surprisingly, the placement of the criterion becomes important in the variance theory because there is a nonlinear transformation when the nodes are activated. This nonlinearity makes $d^{\prime}$ dependent on the activation threshold in the nodes.

The $d^{\prime}$ was maximized by changing the activation threshold $(T)$ and the percentage of nodes active at encoding $(a)$. The maximum $d^{\prime}$ was 2.40 when $T=0.81$ and $a=.052$. Figure 9B plots $d^{\prime}$ as a function of the activation threshold $(T)$ when the percentage of nodes active at encoding was fixed at the optimal value ( $a=$ .052 ). The results show that $d^{\prime}$ has an optimal value when the activation threshold is set between the old and the new distributions. The variance theory suggests that the threshold should be set to the average of the expected old and expected new net inputs. For the parameter values used here, this value is 0.71 , which is near, but slightly lower than the optimal value of 0.81 (the expected value of the new net input is 0 and the expected value of the old net input is 1.42). Note that this result applies when both $a$ and $T$ are set to the optimal value. If $a$ is set to a nonoptimal value, the optimal value of $T$ may deviate significantly from the one proposed by the theory (e.g., if $a=.5$, the optimal value of $T$ is much larger than the expected value of old net inputs of 1.88).

This analysis emphasizes the importance of setting the activation threshold as suggested by the variance theory. Setting the activation threshold between the old and the new expected net inputs yields not only the mirror effect, but also an optimal performance in the network. Notice that the activation threshold $(T)$ is constant even if the subjects' decision criterion $(C)$ is changed. Therefore $d^{\prime}$ will not change when the decision criterion changes. By changing the decision criterion (rather than the recognition threshold), subjects can maintain an optimal $d^{\prime}$ for different confidence levels.

Optimal usage of the state of activation in the cue pattern. An interesting question is how much information is carried in nodes that are active in the encoded pattern, as compared with inactive nodes. If both active and inactive nodes carry a similar amount of information, it is useful to use all the nodes at retrieval. However, if inactive nodes carry little information in relation to the noise, performance can be improved by using only the information in the active nodes.

The information carried in the nodes depends on the amount of weight changes, which, in turn, depends on the percentage of active nodes at encoding $(a)$. For $a=1 / 2$, the absolute values of the weight changes are the same for active and inactive nodes (however, the signs of the weight changes are different). Thus, inactive and active nodes carry the same amount of information, and the performance is optimal when information in both active and inactive nodes is used. For a small $a$, the weight changes are larger for active nodes (proportional to $1-a$ ) than for inactive nodes (proportional to $a$ ). For a sufficiently small $a$, the noise in inactive nodes will overwhelm the information in the weight changes, so that if the information is combined, the inactive nodes will harm the information in the active nodes and performance is optimal if only information from active nodes is used.

The performance of the variance theory was calculated by using the information in all the nodes. This is done by counting the number of nodes that are retrieved to the correct state of activation (i.e., the same state as that during encoding). The mathematical details of this calculation are described at the end of the Appendix. The results are shown by the dotted line in Figure 9A, using the same set of parameters as when $d^{\prime}$ was calculated by using only active nodes, shown by the solid line. The results show that the highest $d^{\prime}$ is found when the decision is based only on active nodes and when $a$ is low. Including inactive nodes in decision lowers $d^{\prime}$. However, for a larger $a$ (above .15 for the parameters used here), it is beneficial to base the decision on all the nodes.

Optimal placement of the recognition criterion for the two classes of items. The recognition criterion $(C)$ does not affect $d^{\prime}$, but it influences performance, as measured by the hit rates and false alarm rates. Therefore, it is necessary to use another criterion for performance 

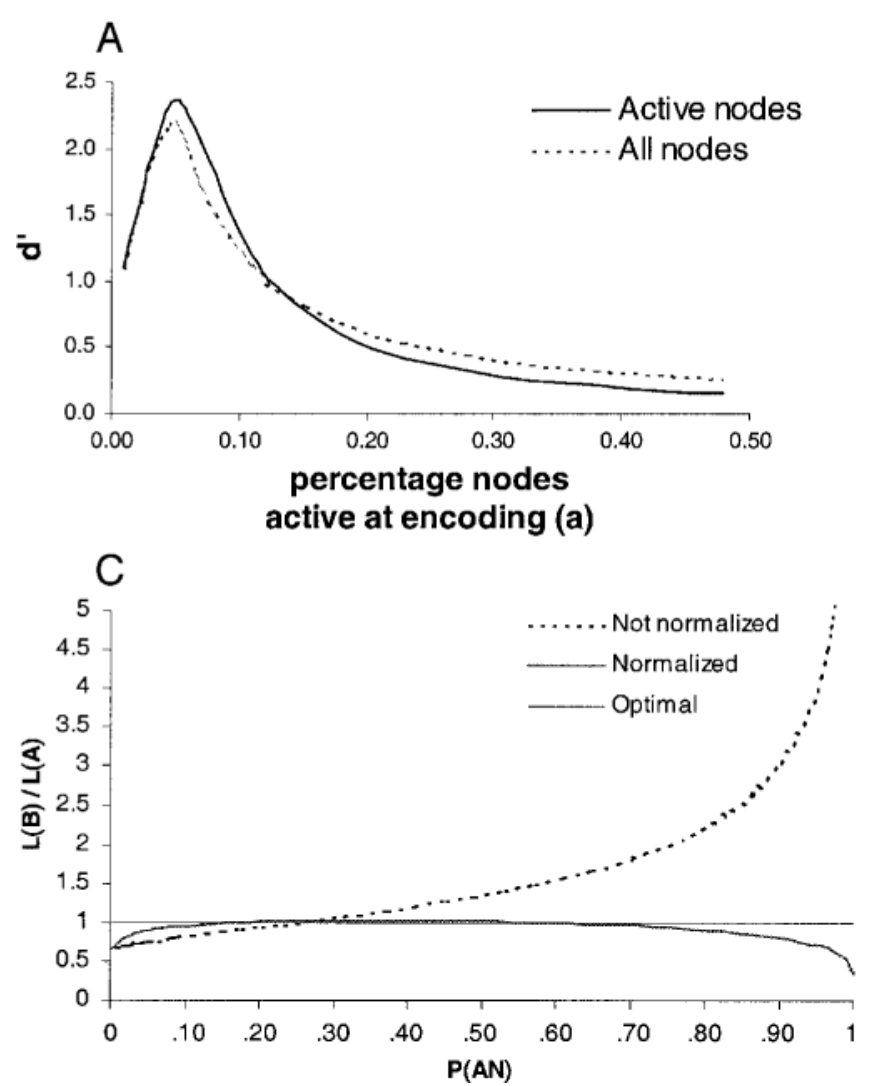

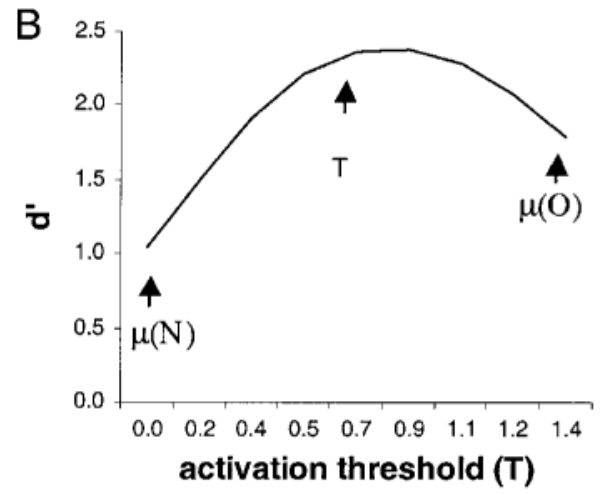

D

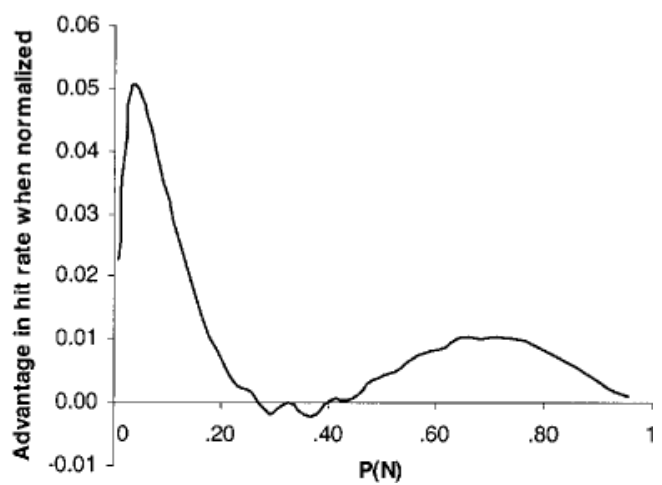

Figure 9. (A) Theoretical $d^{\prime}$ as a function of percentage of nodes active at encoding. The solid line shows the $d^{\prime}$ as a function of percentage of nodes active at encoding when the decision is based only on nodes that are active during encoding. The dotted line shows $d^{\prime}$ when the decision is based on all the nodes. (B) Theoretical $d^{\prime}$ as a function of the activation threshold. The leftmost arrow points at the expected net input of the new items $[\mu(\mathrm{N})]$, the rightmost arrow points at the expected net input of the old items $[\mu(O)]$, and the middle arrow points at the point at the placement of the activation threshold of the nodes. Note that the activation threshold is slightly lower than the optimal point. (C) Optimal placement of the recognition criterion for the two classes. The $y$-axis shows the maximum likelihood for Class B divided by the maximum likelihood for Class A. An optimal performance is found when this ratio is one. The $x$-axis shows the false alarm rate for Class A. The straight line shows the ratio for theoretical optimal performance, the dotted line the ratio before normalization, and the solid curved line the ratio after normalization. See the text for details. (D) The advantage of normalization for different recognition criteria. The $y$-axis shows the total hit rate after normalization minus the total hit rate before normalization as a function of the total false alarm rate on the $\boldsymbol{x}$-axis. See the text for details.

with respect to the placement of the recognition criterion. A natural choice for performance in this context is the probability of hits minus the probability of false alarms. This measurement corresponds to optimal performance when old correct responses and new correct new responses are rewarded equally. It is easy to see that if the standard deviations of the old and the new distributions are equal, the optimal performance will be found if the recognition criterion is set exactly between the distributions. For unequal standard deviations, the optimal recognition criterion is shifted from the midpoint toward the distribution with the smallest standard deviation. More exactly, the optimal recognition criterion is the point at which the old and the new distributions intersect. It is easy to see that this is true, because if the recognition criterion is moved to the left of this point, the rate of increase in false alarms is larger than the rate of increase in hits and performance suffers. If the recognition criterion is moved to the right of this point, the rate of decrease in hits is larger than the rate of decrease in false alarms and performance also suffers (see, e.g., Figure 4D). Formally, $f[S(\mathrm{O})]$ denotes the density of recognition strength of the old distribution, and $f[S(\mathrm{~N})]$ the density of the recognition strength of the new distribution. The ratio between these variables is called the likelihood ratio, $L=f[S(\mathrm{O})] / f[S(\mathrm{~N})]$, and the optimal performance occurs when this ratio is equal to one $(L=1)$.

In the mirror effect, there are two classes of items, each having a new and an old distribution with different standard deviations. The question of optimal performance is complicated by the possibility of using different criteria for the two classes. The performance may then vary, depending on the choice of the two criteria and on additional restrictions on the overall level of confidence. For example, if one class is very easy (i.e., perfect discrimination) and one class is very difficult (i.e., no 
discrimination) and subjects are instructed to respond yes only when they are absolutely certain that they are correct, it may be optimal to set a very high criterion for the difficult class, so that no yes responses will be made for the difficult class, and a moderate criterion for the easy class, so that some yes responses will be made for the easy class. Therefore, any model that optimizes performance for the two classes must combine the criteria for each class so that the performance for the sum of the classes will be optimal.

This problem may formally be stated as follows. Given two classes (A and $\mathrm{B}$ ) with a fixed total false alarm rate $[P(\mathrm{AN})+P(\mathrm{BN})]$, how should the recognition criteria for the two classes $[T(\mathrm{~A})$ and $T(\mathrm{~B})]$ be chosen so that the hit rates are maximized $[P(\mathrm{AO})+P(\mathrm{BO})]$ ? The solution to this problem is surprisingly simple. The optimal performance occurs when the placements of the maximum likelihoods of the two classes are equal:

$$
\begin{aligned}
L(\mathrm{~A}) & =f[S(\mathrm{AO})] / f[S(\mathrm{AN})]=L(\mathrm{~B}) \\
& =f[S(\mathrm{BO})] / f[S(\mathrm{BN})] .
\end{aligned}
$$

It is easy to see that this criterion must be satisfied for optimal performance, because any shift from this point diminishes performance. For example, if the recognition threshold for class A is diminished, the recognition criterion for class B must be increased to keep the total false alarm rate constant. According to the formulation of the problem, the change in total false alarm rates must be equal: $f-f[S(\mathrm{BN})=0]$. The maximum-likelihood ratios are monotonically increasing functions of the recognition criteria; therefore, $L(\mathrm{~A})-L(\mathrm{~B})<0$ when the recognition criteria are changed as specified above. Thus, $L(\mathrm{~A})=f[S(\mathrm{AO})] / f[S(\mathrm{AN})]<f[S(\mathrm{BO})] / f[S(\mathrm{BN})]=$ $L(\mathrm{~B})$, or $f[S(\mathrm{AO})]-f[S(\mathrm{BO})]<0$. This shows that the change in the placement of the criteria from $L(\mathrm{~A})=L(\mathrm{~B})$ results in an overall decrease in hit rate- $(f[S(\mathrm{AO})]-$ $f[S(\mathrm{BO})]<0)$-and performance suffers.

Note that the variance theory only has one overall recognition criterion. However, the theory, and any theory of the mirror effect, specifies how this criterion changes when it is moved over the two classes. Thus, the second criterion can indirectly be inferred from the formulation of the theory. This is done in the variance theory by the normalization factor that scales the recognition differently between the two classes of words.

The intriguing question here is whether the variance theory is optimal or almost optimal in terms of placement of the recognition criterion for the two classes. Figure $9 \mathrm{C}$ plots the maximum likelihood of class $\mathrm{B}$ divided by the maximum likelihood of class $\mathrm{A}[L(\mathrm{~B}) / L(\mathrm{~A})]$ on the $y$-axis. The $x$-axis shows the probability of false alarms for class A when the recognition criteria are changed. The optimal ratio of the maximum likelihood on the $y$-axis is one, and it is plotted as the dotted straight line. The results before normalization (i.e., by counting the number of nodes above the recognition criterion) are plotted in the dotted monotonically increasing line. The results after normalization (i.e., the percentage of active nodes minus the expected number of active nodes divided by the standard deviation of the net input) are plotted as the solid line.

The figure clearly shows that performance before normalization is far from optimal. For a conservative recognition criterion, or low false alarm rates, the maximum likelihood for class A is larger than the maximum likelihood for class B. Therefore, a more liberal criterion for class B and a more strict criterion for class A would be more advantageous. For a liberal recognition criterion, or a high false alarm rate, the maximum likelihood for class B is larger than the maximum likelihood for class A. Therefore, a more liberal criterion for Class A and a stricter criterion for Class B would be beneficial. The only point at which the performance is optimal is when the recognition criterion is unbiased. At this point [around $P(\mathrm{AN})=.25]$, the maximum-likelihood ratio is one.

Normalization improves performance significantly, so the maximum-likelihood ratio is one, or almost one, for a range of criteria. For false alarm rates larger than 0.15 and smaller than 0.60 , the ratio is within two percentage points of one. The maximum likelihood for Class A is larger than that for Class B for false alarm rates less than 0.15 and for false alarm rates larger than 0.60. Thus, there is still some deviation from optimal performance even after normalization. However, the maximum-likelihood ratio is closer to the optimal value for all false alarm rates after normalization than before normalization. Arguably, normalization improves performance sufficiently, so that performance may be described as being near an optimal value for a wide range of recognition criteria.

Overall performance after normalization can be directly compared with performance before normalization. Figure 9D plots the total hit rate after normalization minus the total hit rate before normalization for different total false alarm rates. In this figure, the standard deviation of the net inputs to Class B was set to a 3 (rather than 1.56) in order to make the difference between performance before and after normalization more salient. All other parameters were identical to those in Figure 4D. Furthermore, it is assumed that the number of items in Class A is equal to the number of items in Class B. The total hit rate is equal to the average hit rate of Class $\mathrm{A}$ and Class $\mathrm{B}$, and the total false alarm rate is equal to the average false alarm rate in Class A and Class B.

For all recognition criteria, or for all false alarm rates, performance is better or equal after normalization, as compared with performance before normalization. For an unbiased recognition criterion or a slightly larger recognition criterion, performance is approximately equal before and after normalization [i.e., for $.25<P(\mathrm{~N})<$ $.40]$. For conservative recognition criteria $[P(\mathrm{~N})<.25]$, there is a large advantage for normalized performance over nonnormalized performance. The largest advantage is approximately 0.05 , and it occurs when the false alarm 
rate is approximately 0.05 . For liberal recognition criteria $[P(\mathrm{~N})>.40]$, there is also an advantage for normalized performance. The largest advantage is around 0.01 , and it occurs when the false alarm rate is 0.70 . The advantage for liberal criteria is smaller than the advantage for conservative criteria because of a ceiling effect for large false alarm rates and large hit rates.

\section{A Nonoptimal Network is Inconsistent With Empirical Data of Recognition Memory}

To summarize, it has been shown that performance is optimal when (1) the percentage of nodes active at encoding is low, (2) the activation threshold is set between the new and the old distributions, (3) only nodes active at encoding are used for measuring the recognition strength, and (4) the recognition strength is normalized with the standard deviation of the net input. It is interesting to note that all these conditions are necessary for producing the empirically found memory phenomena.

1. The percentage of active nodes has to be low for producing appropriate standard deviations for the old and the new recognition distributions. If the percentage of active nodes is too high, the standard deviation of the old distribution will approach the standard deviation of the new distribution.

2 . The model predicts the mirror effect only if the activation threshold is set between the old and the new distributions. If the recognition threshold is larger than the expected value of the net input of the old distribution, the hit rate of the easy class will be less than the hit rate of the difficult distribution. Similarly, if the recognition threshold is lower than the expected new net input, the false alarm of the easy class will be larger than the false alarm rate of the difficult class. This is inconsistent with the empirical data for unbiased responses.

3. Assume that the recognition strength is based on all the nodes (i.e., not only nodes inactive during encoding)-for example, by counting the number of nodes in the correct state of activation. This measurement of recognition strength will have at least $50 \%$ of the nodes in the correct state (i.e., $P_{c}>.50$ ) even if the subjects were merely guessing on new items. This would lead to the incorrect prediction that the old recognition strength variance should be smaller than the new recognition strength variance, because $P_{c}\left(1-P_{c}\right) / N$ decreases for $P_{c}$ over .50. This is inconsistent with the finding that the variance of the old distribution is larger than the variance of the new distribution.

4. If the recognition strength is not normalized with the net input, the variance of the recognition strength of the new easy class (AN) will be smaller than the variance of the recognition strength of the new difficult class (BN; see Figure 4C). This is inconsistent with the empirical data.

This analysis indicates that several recognition memory phenomena fall out as a consequence of optimizing performance in the network. If the model is optimized in terms of performance, the model reproduces the empirical data. If the model is made nonoptimal, the model does not reproduce the empirical data. Arguably, the human brain has evolved to optimize storage capacity, and therefore, these memory phenomena occur.

\section{GENERAL DISCUSSION}

This paper has suggested a variance theory for the mirror effect that also applies to the ROC curves. The model is remarkably simple. It has been shown that a two-layer recurrent network, where one layer represents context and one layer represents items, shows these phenomena if performance is measured by counting the number of nodes active at recognition in a way that optimizes performance. The structure of the theory guarantees that high-frequency items have a larger variance in the net inputs than do low-frequency items, because encoding the same item in different contexts increases the variance, whereas the expected net inputs are the same. The theory predicts the mirror effect when the same amount of attention is paid to both classes of stimuli. The standard deviation of the recognition strength is larger for old than for new items because more nodes are active in old items. The standard deviation for the easy class is larger than the standard deviation for the difficult class because the recognition strength is normalized with the standard deviation for the net input.

There are several reasons why the variance theory is interesting. First, the theory is extremely simple. The only necessary assumptions are that recognition is based on recurrent associations between contexts and items and performance is measured by counting the number of nodes in an optimal way. Second, these assumptions are consistent with what is known about how the brain works. Therefore, the model is biologically plausible. Third, the model accounts for a large amount of data, including the mirror effect, exceptions from the mirror effect, ROC curves, list-length effects, and so on. Fourth, the model fits the empirical data well. Fifth, it is easy to implement the model in a connectionist network.

Paying more attention to one of the classes violates the assumption of equal expected net inputs to the two classes. The variance theory predicts that attention to the more difficult class primarily affects the hit rates, whereas the false alarm rates and standard deviations of the underlying distributions are less affected. An experiment supported the prediction. A standard mirror effect was found when attention was divided equally between high- and low-frequency words. However, focusing the subjects' attention on the high-frequency words, either by the presentation frequency or the presentation time, made the hit rate larger for the high-frequency words than for the low-frequency words. This manipulation of attention did not influence the false alarm rates, which were higher for the high-frequency words in all the conditions. Thus, no mirror effect was found when attention was paid to the high-frequency words. Nor did the focusing of attention influence the order of the standard deviations. The standard deviations were larger for the low-frequency distri- 
bution than for the high-frequency distribution. This was true for the new and the old distributions both when attention was paid to high-frequency words and when attention was divided equally between the two classes (except in the new frequency control condition, where the standard deviations were equal).

The variance theory predicts the order of the standard deviations of the underlying distributions for the following reasons. The standard deviations of the old distributions are predicted to be larger than those of the new distributions because more nodes are activated in the old distributions. The standard deviations of the easy class distributions are predicted to be larger than the standard deviations of the difficult class distributions because the recognition strength is normalized by the item's difficulty estimated from the standard deviation of the net inputs. This is consistent with the empirical data.

In contrast, the attention-likelihood theory does not constrain the old distribution to be larger than the new distribution for the difficult class (it can be larger or smaller, depending on parameter settings). The variance theory allows the following two orders of the standard deviations: $\sigma_{s}(\mathrm{BN})<\sigma_{s}(\mathrm{BO})<\sigma_{s}(\mathrm{AN})<\sigma_{s}(\mathrm{AO})$ and $\sigma_{s}(\mathrm{BN})<\sigma_{s}(\mathrm{AN})<\sigma_{s}(\mathrm{BO})<\sigma_{s}(\mathrm{AO})$. The first order is the more common, although the second order occurs occasionally (see, e.g., Glanzer et al., 1993, Experiment 1). In addition, the attention-likelihood theory allows $\sigma_{s}(\mathrm{BO})<\sigma_{s}(\mathrm{BN})<\sigma_{s}(\mathrm{AN})<\sigma_{s}(\mathrm{AO})$, according to Equation 2, which to the author's knowledge has not been found in empirical data.

The variance theory predicts that strength variables, such as study time, repetition, and study instructions, affect the expected net input. For example, increasing study time will increase the net input that improves the hit rate. Increasing the net inputs also causes an increase in the activation threshold that diminishes the false alarm rates.

The variance theory has no (i.e., lateral) connections within the item layer, and there are no connections within the context layer. Including intraitem connections in recognition makes it impossible to tell whether the recognition strength comes from encoding during the learning episode or from another preexperimental learning episode. Thus, there would be a confounding between the item's frequency and recognition strength. For example, if the recognition strength in the variance theory included intraitem connections and used a constant recognition criterion, it would predict a higher hit rate and a higher false alarm rate for high-frequency items than for low-frequency items. Thus, the hit rate for highfrequency words would be larger than that for lowfrequency words, which is contrary to the data on the mirror effect. This issue is called the frequency/recognitionstrength confounding problem. Other models may be vulnerable to this problem, depending on their specific assumptions. The variance theory is immune from this problem, because recognition strength is based on the association between the context and the item that yields a pure measurement of the strength of the target in a par- ticular episode. Net inputs within the item population are not used, because these connections are highly correlated with the frequency of the item.

This frequency/recognition-strengthconfounding problem may be relevant to several distributed models that assume that recognition strength increases with frequency, thus making the false alarm rate higher for high- than for low-frequency stimuli. This is often implemented in distributed models by including intraitem associations in the measurement of recognition strength. Thus, when intraitem and item-context associations are added, it is not possible to know whether the intraitem strength occurs because an item has been encoded in the to-beretrieved-from list or at another episode.

Although the intraitem associations are not used to measure recognition strength, they may play an important role in recognition. In the first step of recognition, these associations may be used for deblurring unclear information in the item cue (a similar mechanism occurs for the context cue). Arguably, this deblurring mechanism works well for well-known words; however, for nonwords, it is much more likely to fail. Such failure will activate features that were not active in the encoded representation. It is more likely that these wrongly activated nodes represent high-frequency features, because it is more likely to converge on high-frequency features. There are two interesting implications of this perspective. First, the wrongly activated nodes will use the wrong connections between the context and the item. Second, because the wrongly activated nodes represent high-frequency features, the average variability will be larger for nonwords than for words. This is a plausible account of the poor recognition performance with nonwords, as compared with words. It is also a tentative account of why nonwords can be seen as a difficult class with a higher variability than that for words in the variance theory. However, further work is needed before any firm conclusion can be made regarding this aspect of the theory.

A problem similar to frequency/recognition-strength confounding occurs if within-context connections are used at recognition. If the context is temporally correlated, the within-context connections are influenced by list length. This causes a confounding between list length and recognition strength. This issue is called the listlength/recognition-strength confounding problem. Other models may be vulnerable to this problem, depending on their specific assumptions.

Another issue is whether the variance theory can account for the mirror effect found in abstract and concrete words, where words from a concrete class are more easily discriminated than words from an abstract class. The variance theory can account for this, given the assumption that the variance of the net input is larger for abstract than for concrete words. However, at this point it is not completely clear how this assumption can be motivated. A possibility is that although these two classes are equated for word frequency, the contexts associated with an abstract word are more variable than the contexts as- 
sociated with a concrete word. This larger variability in context for abstract words may lead to a larger variability in the net input. Another possibility is that the active features in abstract words are more general and, therefore, associated with more contexts. Nodes active in concrete words may represent more specific features, activated with a lower frequency, and therefore associated with fewer contexts. Thus, features in abstract words may be of a higher frequency than features in concrete words, although the frequencies of the items are the same. This would lead to a mirror effect in the model. However, at this point, no claim is made that variance theory can handle this phenomenon.

The variance theory's account of the list-length and list-strength effects is arguably much simpler than the subjective-likelihood's account. The activation threshold is set so that, on average, half of the nodes active during encoding are active during recognition. The activation threshold is constant within one condition but may change between conditions-for example, when study time is changed. Furthermore, subjects do not need to estimate the list length and the probability that a particular item is drawn from the study list.

The variance theory has advantages over the attentionlikelihood theory. As was discussed in more detail above, the attention-likelihood theory requires subjects to classify the stimulus. Depending on this classification, the subjects must know (consciously or unconsciously) how much attention is paid to the stimuli in order to calculate the log-likelihood ratios. Thus, the yes-no decision is based on the subjects' classification of the stimuli. The variance theory does not require a classification of the stimuli. During the calculation of recognition strength, the standard deviation of the net input of the item is used $\left(\sigma_{h}^{\prime}\right)$, so the subject does not need to know the class or the standard deviation of the class $\left(\sigma_{h}\right)$. The increase in the hit rate and decrease in the false alarm rate for the easier class occurs, according to the theory, because the variance is smaller for the easier class. The variance theory has a simple mathematical base; subjects count the number of activated nodes minus the expected value divided by the standard deviation of the net input of the item. An explicit solution is presented that requires only calculating the probabilities of two $z$-transformations.

The subjective-likelihood theory uses feature content of the items for addressing issues regarding item similarity and word frequency. In particular, high-frequency words are assumed to have a higher noise, or variability, than do low-frequency words. The variance theory also uses variability that depends on frequency. However, the variance theory simulates the increase in variance during each presentation of a feature in different contexts, thus making it an unavoidable phenomenon for high-frequency features. In the subjective-likelihood theory, the feature variance is introduced as an assumption, or a constant, and it is not explicitly simulated.

There are several other differences between the variance theory and the subjective-likelihood theory. The subjective-likelihood theory is based on log-likelihood ratios. In the variance theory, log-likelihood ratios are not necessary to account of the mirror effect and for $z$ ROC curves. Instead, the variance theory uses the number of active nodes normalized by the variance of the net input as the measurement of recognition strength.

Another difference is the use of one detector for each item in the subjective-likelihood theory. This makes the model essentially local, whereas the variance theory is distributed. This property may cause difficulties in implementing the model in a connectionist network. However, see McClelland and Chappell (1998) for a brief discussion of this topic. An implementation of the variance theory is straightforward.

\section{REFERENCES}

Feller, W. (1968). An introduction to probability theory and its application. New York: Wiley.

Gillund, G., \& Shiffrin, R. M. (1984). A retrieval model for both recognition and recall. Psychological Review, 91, 1-67.

Glanzer, M., \& AdAMS, J. K. (1985). The mirror effect in recognition memory. Memory \& Cognition, 13, 8-20.

Glanzer, M., \& AdAMS, J. K. (1990). The mirror effect in recognition memory: Data and theory. Journal of Experimental Psychology: Learning, Memory, \& Cognition, 16, 5-16.

Glanzer, M., Adams, J. K., \& Kim, K. (1993). The regularities of recognition memory. Psychological Review, 100, 546-567.

Glanzer, M., \& Bowles, N. (1976). Analysis of the word frequency effect in recognition memory. Journal of Experimental Psychology: Human Learning \& Memory, 2, 21-31.

Glanzer, M., KisoK, K., \& ADAms, J. K. (1998). Response distributions as an explanation of the mirror effect. Journal of Experimental Psychology: Learning, Memory, \& Cognition, 24, 633-644.

GREENE, R. L. (1996). Mirror effect in order and associative information: Role of response strategies. Journal of Experimental Psychology: Learning, Memory, \& Cognition, 22, 687-695.

Hertz, J., Krogh, A., \& Palmer, R. G. (1991). Introduction to the theory of neural computation. Reading, MA: Addison-Wesley.

HintZMAn, D. L. (1988). Judgment of frequency and recognition memory in a multiple trace memory model. Psychological Review, 95, 528-551.

Hopfield, J. J. (1982). Neural networks and physical systems with emergent collective computational abilities. Proceedings of the $\mathrm{Na}$ tional Academy of Sciences, 79, 2554-2558.

HopfiEld, J. J. (1984). Neurons with graded response have collective computational properties like those of two-state neurons. Proceedings of the National Academy of Sciences, 81, 3088-3092.

Humphreys, M. S., Bain, J. D., \& Pike, R. (1989). Different way to cue a coherent memory system: A theory for episodic, semantic and procedural tasks. Psychological Review, 96, 208-233.

KIM, K., \& GLAnZER, M. (1993). Speed versus accuracy instructions, study time, and the mirror effect. Journal of Experimental Psychology: Learning, Memory, \& Cognition, 19, 638-652.

KRUSCHKE, J. K. (1992). ALCOVE: An exemplar-based connectionist model of category learning. Psychological Review, 99, 22-44.

KuČera, H., \& Francis, W. N. (1967). Computational analysis of present-day American English. Providence, RI: Brown University Press.

LEWANDOWSKY, S. (1991). Gradual unlearning and catastrophic interference: A comparison of distributed architectures. In W. E. Hockley \& S. Lewandowsky (Eds.), Relating theory and data: Essays on human memory in honor of Bennet B. Murdock (pp. 445-476). Hillsdale, NJ: Erlbaum.

Li, S.-C., \& Lindenberger, U. (1999). Cross-level unification: A computational exploration of the link between deterioration of neurotransmitter systems and dedifferentiation of cognitive abilities in old age. In L.-G. Nilsson \& H. J. Markowitsch (Eds.), Cognitive neurosciences of memory (pp. 103-146). Seattle: Hogrefe \& Huber. 
Li, S.-C., Lindenberger, U., \& Frensch, P. A. (2000). Unifying cognitive aging: From neuromodulation to representation to cognition. Neurocomputing, 32-33, 879-890.

McClelland, J. L., \& Chappell, M. (1998). Familiarity breeds differentiation: A subjective-likelihood approach to the effects of experience in recognition memory. Psychological Review, 105, 724-760.

Metcalfe, J. (1982). A composite holographic associative recall model. Psychological Review, 89, 627-658.

MuRdock, B. B. (1982). A theory for the storage and retrieval of item and associative information. Psychological Review, 89, 609-626.

Murdock, B. B. (1998). The mirror effect and attention-likelihood theory: A reflective analysis. Journal of Experimental Psychology: Learning, Memory, \& Cognition, 24, 524-534.

OKADA, M. (1996). Notions of associative memory and sparse coding. Neural Networks, 9, 1429-1458.

Ratcliff, R., Sheu, C.-F., \& Gronlund, S. D. (1992). Testing global memory models using ROC curves. Psychological Review, 99, 518-535.

Rumelhart, D. E., Hinton, G. E., \& Williams, R. J. (1986). Learning representation by backpropagating errors. Nature, 323, 533-536.

Shiffrin, R. M., \& Steyvers, M. (1997). A model for recognition memory: REM-retrieving effectively from memory. Psychonomic Bulletin \& Review, 4, 145-166.

SIKSTRÖM, S. (1996a). TECO: A connectionist theory of successive episodic tests. Umeå: Doctoral dissertation, Umeå University (ISBN 91-7191-155-3).

SiKST Röm, S. (1996b). The TECO connectionist theory of recognition failure. European Journal of Cognitive Psychology, 8, 341-380.

Sikst RöM, S. (1999). Power function forgetting curves as an emergent property of biologically plausible neural networks model. International Journal of Psychology, 34, 460-464.

Stretch, V., \& WiXted, J. T. (1998a). Decision rules for recognition memory confidence judgments. Journal of Experimental Psychology: Learning, Memory, \& Cognition, 24, 1397-1410.

Stretch, V., \& Wixted, J. T. (1998b). On the difference between strength-based and frequency-based mirror effects in recognition memory. Journal of Experimental Psychology: Learning, Memory, \& Cognition, 24, 1379-1396.

\section{NOTE}

1. The expected number of nodes active at recognition is $a / 2$, given that the standard deviations of the percentages of active nodes for new $\left[\sigma_{p}(\mathrm{~N})\right]$ and old items $\left[\sigma_{p}(\mathrm{O})\right]$ are equal. However, if the standard deviations are different, the expected number of active nodes will be

$$
\frac{\sigma_{p}(\mathrm{~N})}{\sigma_{p}(\mathrm{~N})+\sigma_{p}(\mathrm{O})} a
$$

Because the new variance is smaller than the old variance, this value will be slightly less than $a / 2$, typically around $0.44 a$ if the ROC curve is 0.8 . It is actually more precise to use this value. In this paper, the approximation $a / 2$ is used, except that in the simulations, where the expression above is used.

\section{APPENDIX}

\section{The Expected Value of the Net Input and the Variance of the Net Input}

Inserting the weight changes into the net input solves the expected value of the net input for old items. In this Appendix, the superscripts representing the item layer (d) and the context layer (c) are dropped. Only the active states of activation contribute to the net input, so $\xi_{j}=\xi_{j}=1$ :

$$
\mu_{h}(\mathrm{O})=\sum_{j}^{N} \Delta \mathrm{w}_{i j} \xi_{j}=\sum_{j}^{N}\left(\xi_{i}-a\right)\left(\xi_{j}-a\right) \xi_{j}=a(1-a)^{2} N
$$

The expected value of the net inputs for the new items is zero:

$$
\mu_{h}(\mathrm{~N})=0 .
$$

The expected value of the weights is zero. The variance of the net input is calculated by squaring each term in the net input. Let $P$ represent the number of patterns stored in the network. If the patterns are uncorrelated or a new item is presented, the variance of the net input is

$$
\begin{aligned}
\sigma_{h}^{2}(N)= & \sum_{j}^{N}\left(\mathrm{w}_{i j} \xi_{j}\right)^{2}=\sum_{i}^{N} \sum^{P}\left\{\left(\xi_{i}-a\right)\left(\xi_{j}-a\right) \xi_{j}\right\}^{2} \\
= & \left\{[(1-a)(1-a)]^{2} a^{2}+2[(0-a)(1-a)]^{2} a(1-a)\right. \\
& \left.+[(0-a)(0-a)]^{2}(1-a)^{2}\right\} a P N=a^{3}(1-a)^{2} P N
\end{aligned}
$$

For an old item, the variance of the net input to the context layer from the item layer depends on the frequency of the item $(f)$. All the $a N$ weights supporting a context node contribute to the net input in the same direction:

$$
\begin{aligned}
\sigma_{h}^{2}(f) & =f\left(\sum_{j}^{N} \mathrm{w}_{i j} \xi_{j}\right)^{2}=f\left[\sum_{i}^{N}\left(\xi_{i}-a\right)\left(\xi_{j}-a\right) \xi_{j}\right]^{2} \\
& =a^{4}(1-a)^{2} f N^{2} .
\end{aligned}
$$

The same function can be used for calculating the variance of the net input to a node in the item layer when the same context is associated with several items. Let the same context be associated with $L$ items in a list. Furthermore, let the context between different lists be uncorrelated. The variance of the net input to an item node is

$$
\sigma_{h}^{2}(f)=a^{4}(1-a)^{2} L N^{2} .
$$

The expected variance for all nodes is the weighted sum of these two terms. Half of the nodes are context nodes, and half of the nodes are item nodes. Therefore, the expected variance of the net input to all nodes, given an item with a frequency of $f$ and a list length of $L$ in a network where $p$ patterns have been encoded, is

$$
\sigma_{h}^{2}(\mathrm{O})=\{(f+L) a N / 2+p\} a^{3}(1-a)^{2} N
$$

\section{Performance in the Variance Model When All Nodes Are Used}

In the variance model, recognition strength is based on nodes active at encoding. However, if recognition strength is based on all the nodes, performance can be calculated as follows. The $d^{\prime}$ is calculated by using Equation 8 , where $P_{c}(\mathrm{O})$ and $P_{c}(\mathrm{~N})$ are the number of correct nodes. The number of correct nodes is the number of nodes active at retrieval that also is active at encoding (i.e., calculated as usual with Equation 7) plus the number of inactive nodes at encoding that also are inactive at retrieval. The latter value can be calculated by replacing $a$ with $1-a$ in Equation 7 and using the expected value of old net inputs for inactive nodes, $a^{2}(1-a) N($ compare with Equation A1).

(Manuscript received February 9, 1999; revision accepted for publication October 30, 2000.) 